Aus der Klinik für Klinische Neurophysiologie

(Prof. Dr. med. W. Paulus)

der Medizinischen Fakultät der Universität Göttingen

\title{
Neuroplastische Effekte transkranieller \\ Nah-Infrarot-Stimulation \\ unterschiedlicher Stimulationsdauer auf die \\ kortikale Exzitabilität
}

\author{
INAUGURAL - DISSERTATION \\ zur Erlangung des Doktorgrades der \\ Medizinischen Fakultät der
}

Georg-August-Universität zu Göttingen

vorgelegt von

Anna Sophie Jakob

aus

Rodewisch

Göttingen 2017 
Dekan:

Prof. Dr. rer. nat. H. K. Kroemer

Referentin:

Prof. Dr. Andrea Antal

Ko-Referentin: Prof. Dr. Melanie Wilke

Drittreferentin: Prof. Dr. Margarete Schön

Datum der mündlichen Prüfung: 14.03.2018 
Hiermit erkläre ich, die Dissertation mit dem Titel "Neuroplastische Effekte transkranieller Nah-Infrarot-Stimulation unterschiedlicher Stimulationsdauer auf die kortikale Exzitabilität" eigenständig angefertigt und keine anderen als die von mir angegebenen Quellen und Hilfsmittel verwendet zu haben.

Göttingen, den 30.11.2017 


\section{Inhaltsverzeichnis}

1. Einleitung und Grundlagen

$1.1 \quad$ Allgemeines

1.2 Transkranielle Laserstimulation.

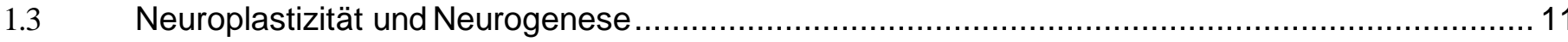

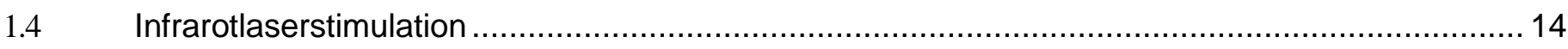

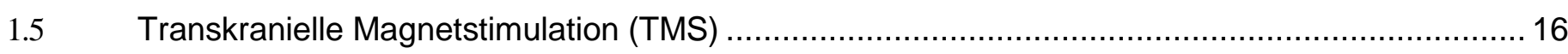

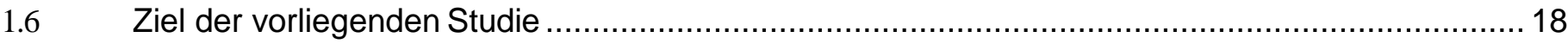

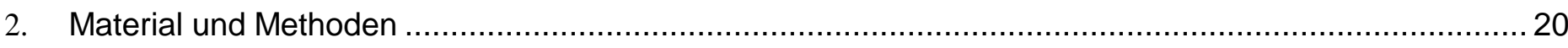

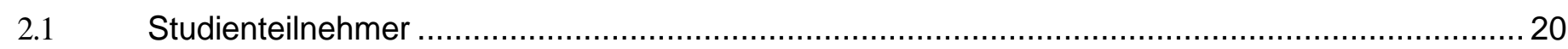

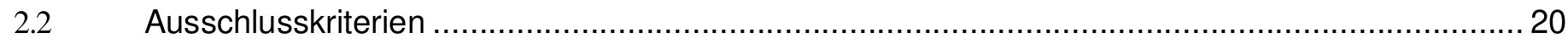

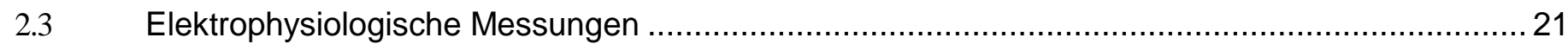

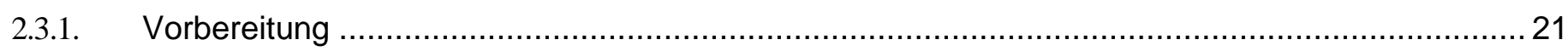

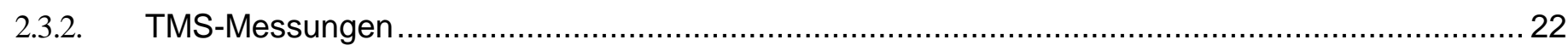

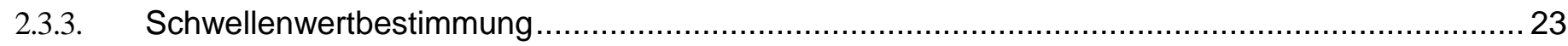

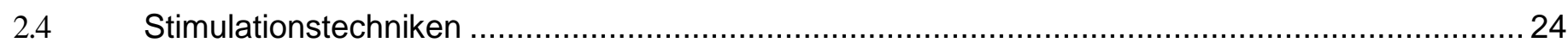

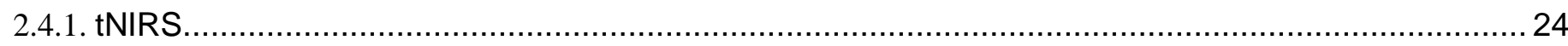

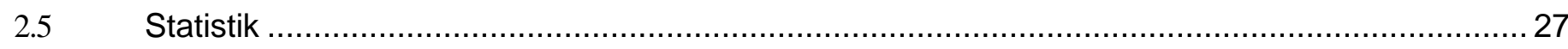

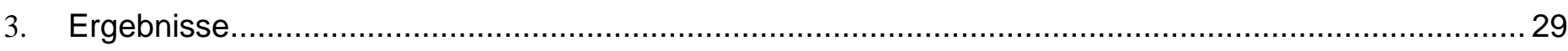

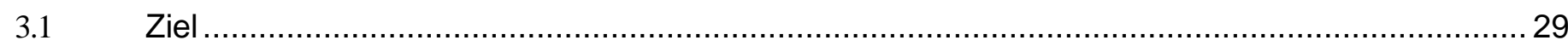

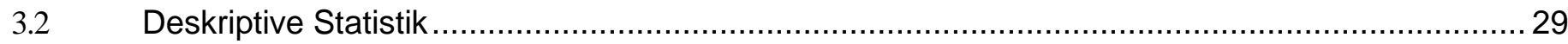

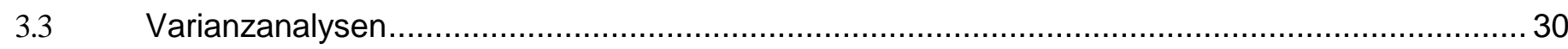

3.3.1. Vergleich Stimulation (5 minütige tNIRS und 20minütige tNIRS) und Plazebostimulation............. 30

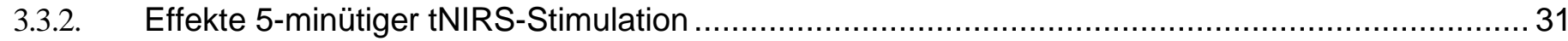

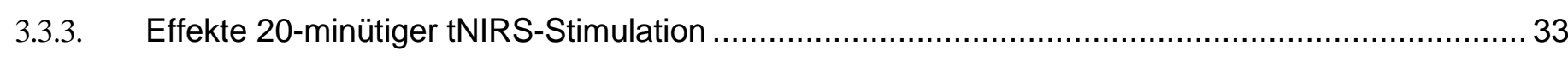

3.3.4. Vergleich 5 - minütige und 20 - minütige Stimulation ................................................ 34

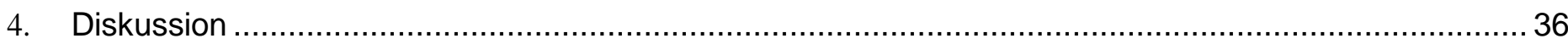

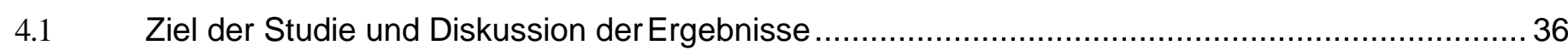

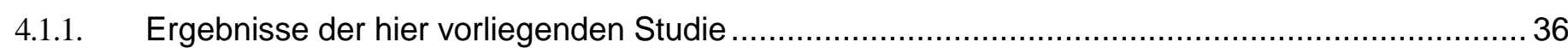

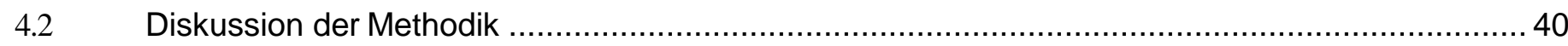

4.3 Diskussion der Zielgrößen im Vergleich zu vorhergehenden Studien ........................................ 42

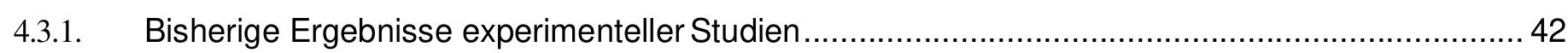

4.3.2. Einsatz der Laser Therapie in klinischen Studien ......................................................... 43

4.3.3. Diskussion der möglichen Ursachen einer Veränderung der kortikalen Exzitabilität ..................... 44

4.4 Veränderungen kortikaler Exzitabilität, hervorgerufen durch andere nicht-invasive Methoden....... 45 
4.4.1. Stimulation durch tDCS (transcranial direct-current stimulation) 45

4.4.2. Abhängigkeit der Effekte von derArt der tDCS-Applikation 46

4.4.3. Abhängigkeit der Effekte von der Dauer dertDCS-Applikation. 47

4.5 Ausblick und Limitationen. 50

5. Zusammenfassung 52

6. Literaturverzeichnis 54 


\section{Verzeichnis der Abkürzungen}

\begin{tabular}{|c|c|}
\hline A & Ampere \\
\hline Abb & Abbildung \\
\hline ADM & Abductor digiti minimi \\
\hline $\mathrm{Ag}$ & Silber \\
\hline $\mathrm{Ag} / \mathrm{Cl}$ & Silberchlorid \\
\hline AMT & active motor threshold \\
\hline ANOVA & $\begin{array}{l}\text { analysis of variance } \\
\text { (Varianzanalyse) }\end{array}$ \\
\hline ATP & Adenosintriphosphat \\
\hline $\mathrm{CCO}$ & Cytochrom - C - Oxidase \\
\hline $\mathrm{Cl}$ & Chlorid \\
\hline EMG & Elektromyographie \\
\hline EPSP & $\begin{array}{l}\text { excitatory postsynaptic potential } \\
\text { (exzitatorisch postsynaptisches Potential) }\end{array}$ \\
\hline FADH & Flavin-Adenin-Dinukleotid \\
\hline FDI & Musculus interosseus dorsalis I \\
\hline GABA & $\begin{array}{l}\text { gamma-aminobutyricacid } \\
\text { (y - Aminobuttersäure) }\end{array}$ \\
\hline $\mathrm{Hz}$ & Hertz \\
\hline I-Welle & $\begin{array}{l}\text { facilitatory indirect wave } \\
\text { (fazilitierende indirekte Welle) }\end{array}$ \\
\hline IPSP & $\begin{array}{l}\text { inhibitory postsynaptic potential } \\
\text { (inhibitorisches postsynaptisches Potential) }\end{array}$ \\
\hline $\mathrm{kHz}$ & kilo Hertz \\
\hline LED & $\begin{array}{l}\text { light emitting diode } \\
\text { (Leuchtdiode) }\end{array}$ \\
\hline LLLT & low-level-laser therapy \\
\hline LTP & $\begin{array}{l}\text { long time potentiation } \\
\text { (Langzeitpotenzierung) }\end{array}$ \\
\hline
\end{tabular}


M1

MEP

$\mathrm{NADH}$

NEST

NILLL

NIR

$\mathrm{nm}$

NMDA

RMT

Tab

tDCS

TMS

tNIRS

tPA

tRNS

W primäre motorische Rinde

motorisch evozierte Potentiale

Nicotinamidadenindinukleotid

the NeuroThera Effectiveness and Safety Trial - study

near - infrared - low - level - laser

near - infrared

(Nahinfrarot)

Nanometer

N-Methyl-D-Aspartat

resting motor threshold

(Ruhemotorschwelle)

Tabelle

transcranial direct current stimulation

(transkranielle direkte Strom Stimulation)

transkranielle Magnetstimulation

transcranial near-infrared stimulation

(transkranielle Nah-Infrarot-Stimulation)

tissue plasminogen activator

transcranial random noise stimulation

(transkranielle Geräuschstimulation)

Watt 


\section{Einleitung und Grundlagen}

\subsection{Allgemeines}

Neurologische Krankheiten sind sehr vielfältig und in unserer Gesellschaft immer häufiger, jedoch stoßen gerade dort konventionelle Behandlungsmöglichkeiten immer wieder an ihre Grenzen. So ist gerade der Schlaganfall nach Angaben der American Heart Association die dritthäufigste Todesursache in Industriestaaten und außerdem führende Ursache für Behinderungen im Erwachsenenalter (Thom et al. 2006), in Europa erleiden jährlich mehr als 600000 Menschen einen Schlaganfall (Litscher und Litscher 2013).

Als einzige klinisch etablierte Behandlungsmöglichkeit gilt heute nach wie vor die medikamentöse Therapie mit Tissue Plasminogen Activator (tPA), welches spätestens drei Stunden nach dem Schlaganfallereignis dem Patienten appliziert werden sollte, um eine therapeutische Wirkung erreichen zu können (The National Institute of Neurological Disorders and Stroke rt-PA Stroke Study Group 1995). Angesichts der großen Anzahl betroffener Menschen in Industrienationen und den mit dem Schlaganfall einhergehenden Behinderungen und Spätfolgen hätte eine Möglichkeit, Patienten auch noch bis zu 24 Stunden nach dem Akutereignis effektiv therapieren zu können große Relevanz. So könnten beispielsweise neurologische Spätfolgen, damit einhergehende Behinderung und erhebliche Lebensqualitätseinschränkung reduziert werden. Dies wäre nicht nur medizinisch und für die betroffene Einzelperson, sondern beispielsweise auch wirtschaftlich von großem Nutzen. Deshalb sind aktuell verschiedene neue invasive sowie nichtinvasive Methoden Gegenstand der Forschung, unter anderem die Therapie 
mittels Laser (Lampl et al. 2007, Lapchak 2010).

Seit der Erfindung des Lasers 1960 und der Erkenntnis, dass diese Technik Möglichkeiten zum klinischen Einsatz birgt und unter anderem zur Verbesserung der Wundheilung und zur Reduzierung von Schmerzen, Entzündung und Schwellung (Mester et al. 1971, Chow et al. 2009 ) dienen kann, wird vermehrt versucht, diese revolutionäre Technik auch zur Therapie neuronaler Krankheiten einzusetzen, um so das Behandlungsspektrum zu erweitern und gegebenenfalls eine Möglichkeit zu erhalten, eine Vielzahl verschiedener Schädigungen, von traumatischen Ereignissen bis hin zu degenerativen Prozessen erfolgreich zu behandeln. Zu diesem Zweck wird das Verfahren der sogenannten transkraniellen Nah-Infrarot Laserstimulation oder auch transcranial near-infrared stimulation (tNIRS) entwickelt und angewendet. Das leistungsschwache Laserlicht der LLLT (low-level-laser therapy), welches im Bereich von 1-1000 Watt arbeitet, ermöglicht so die Auslösung eines biochemischen Prozesses in der Zelle, es treten dabei aber keine thermischen Effekte auf (Hashmi et al. 2010).

\subsection{Transkranielle Laserstimulation}

„Transkranielle Hirnstimulation mit low-level-laser therapy (LLLT) ist der Gebrauch direktionaler Niedrigenergie und stark-fließenden monochromatischen oder quasimonochromatischen Lichts von Lasern oder LEDs im roten- bis infraroten Wellenbereich, um eine neurobiologische Funktion $\mathrm{zu}$ modulieren oder neurotherapeutische Effekte zu induzieren auf eine nicht destruktive und nicht thermale Weise“ (Rojas und Gonzalez-Lima 2013).

Die Sicherheit der transkraniellen Laserstimulation wurde in diversen Tiermodellen belegt 
sowohl in Ratten als auch in Kaninchen und es wurden die jeweiligen sicheren Parameter zur Leistung, Wellenlänge und Dauer der Stimulation festgelegt. (Oron et al. 2006; De Taboada, llic et al. 2006; Lapchak et al. 2007; Lapchak, Wei und Zivin 2004).

In verschiedenen Studien konnte für schwerwiegende neurologische Erkrankungen schon ein therapeutischer Nutzen der low-level-laser therapy nachgewiesen werden. So konnte beispielsweise am Tiermodell gezeigt werden, dass die neurologischen Defizite nach einem Schlaganfall durch Applikation von LLLT reduziert werden konnten (Oron et al. 2006). Hierbei wurde Lasertherapie mit einer Wellenlänge von 808 nm 24 Stunden nach dem ischämischen Ereignis mit $7,5 \mathrm{~mW} / \mathrm{cm}^{2}$ eingesetzt, und eine verbesserte Genesung des Hirngewebes konnte bis zu drei Wochen nach dem Infarktereignis beobachtet werden. Außerdem war die Zahl neu gebildeter Nervenzellen signifikant erhöht.

Eine Studie von Lapchak und De Taboada (2010) konnte weiterhin im Tiermodell die erhöhte ATP-Produktion nach LLLT nachweisen. Dazu wurde Lasertherapie mit einer Wellenlänge von $808 \mathrm{~nm}$ nach embolischen Infarkten in Kaninchen benutzt und es konnte ein Anstieg des ATP-Gehalts sowohl bei kontinuierlicher Laserstrahlung als auch in der pulsatilen Applikation gezeigt werden.

Aber nicht nur im Tiermodell konnte die Effektivität von LLLT demonstriert werden, auch bei menschlichen Schlaganfall-Patienten sind Studien vorhanden, die eine verbesserte und schnellere Regeneration im kognitiven Bereich und in Gedächtnisleistungen belegen (Lampl et al. 2007, Stemer et al. 2010, Zivin et al. 2009). Weiterhin konnte die Effektivität der LLLT auch nach traumatischen Hirnverletzungen gezeigt werden, so konnte beispielsweise im Tiermodell der Nachweis erbracht werden, dass bei Laserapplikation mit $200 \mathrm{~mW}$ bei $808 \mathrm{~nm}$ vier Stunden nach Hirnverletzung das motorische Outcome fünf 
Tage nach dem Trauma- Ereignis signifikant verbessert werden konnte (Oron et al. 2007). Worauf genau die Effektivität der Lasertherapie beruht, konnte schon teilweise erforscht werden. Einer der Mechanismen, auf denen die Wirksamkeit der LLLT beruht, ist die Möglichkeit, die Nervenzellen vor dem Zelluntergang zu bewahren. Dieser Zelltod kann beispielsweise ausgelöst werden durch hypoxische, traumatische oder toxische Insultgeschehnisse (Naeser et al. 2011). So konnte mittels in vitro -Studien beispielsweise die protektive Wirkung der LLLT auf Neuronen nachgewiesen werden, welche Methanol ausgesetzt waren (Naeser et al. 2011, Eells et al. 2003).

\subsection{Neuroplastizität und Neurogenese}

Vermutlich beruht die Effektivität der LLLT auch noch auf einem weiteren Mechanismus, nämlich auf der Anregung der für die Plastizität des Gehirns entscheidenden Neurogenese, also der Neuentstehung neuronaler Zellen und deren Vorstufen (Galvan und Jin 2007). Das Gehirn als Gewebe bietet so die einzigartige Möglichkeit der Neuroplastizität durch Neurogenese und Neuronenmigration, was zum Beispiel Lernvorgänge auch im späten Erwachsenenalter noch ermöglicht (Rossini und Pauri 2000).

An dieser Plastizität des Gehirns sind Mitochondrien als die Kraftwerke der Zelle - und somit für diese überlebensnotwendig - beteiligt. Präsent in allen Zelltypen spielen Mitochondrien deshalb natürlich auch im Zellstoffwechsel des Gehirns, wie beispielsweise in der Zellatmung (Schapira 2012), eine entscheidende Rolle. So gibt es aber auch Erkenntnisse, dass Mitochondrien durch Fehlfunktion entsprechend auch Anteil an Ursachen diverser neurologischer Erkrankungen haben können, wie es 
beispielsweise durch Verlust eines mitochondrialen Transkriptionsfaktors zum vermehrten Auftreten parkinsonoider Phänotypen kommen kann (Ekstrand et al. 2007).

Als Zellorganellen, welche die Atmungskette beinhalten und damit unter anderem die Energieproduktion in Form der ATP-Produktion einer Zelle gewährleisten, sowie durch Regulation verschiedener Mechanismen wie Apoptose, Signalweitergabe und Proteinbildung das Überleben einer Zelle sicherstellen, bilden Mitochondrien zentrale Bestandteile einer Zelle, die allerdings bei Fehlfunktion auch zu Dysfunktion der Zelle beziehungsweise bis hin zu komplexen, unter anderem neurologischen Krankheitsbildern führen können.

Die Mitochondrien konnten als Ort der Wirkung von Laserlicht gezeigt werden (Karu 1999; Passarella et al. 1984; Greco et al. 1989). Mechanismen der Wirkung der LLLT beruhen wahrscheinlich auf der Absorption von monochromalem sichtbarem Licht und der NIR- Strahlung (Nahinfrarot-Strahlung) durch Bestandteile der Atmungskette (Hashmi et al.2010).

Das Innere der Mitochondrienmembran, die Atmungskette, besteht aus fünf verschiedenen Komplexen: der NADH-Dehydrogenase, der Succinat-Dehydrogenase, der Cytochrom-C- Reduktase, der Cytochrom-C-Oxidase und der ATP-Synthase. Durch die frei beweglichen Ubiquinase und Cytochrom-C kommt es zum Elektronentransport zwischen diesen Komplexen. Durch den von der Atmungskette geleisteten Elektronentransfer vom Coenzym Nicotinamidadenindinukleotid (NADH) zu FlavinAdenin-Dinukleotid (FADH) wird ein Protonengradient erzeugt, durch welchen dann Energie frei wird. Als letztlicher Elektronenakzeptor arbeitet schließlich Sauerstoff, welcher in der Atmungskette an den Komplex IV gebunden und zu Wasser umgewandelt wird. 
Bei Zellstress wird durch die Mitochondrien unter anderem Stickoxid gebildet, welches an der Cytochrom-C-Oxidase den Sauerstoff verdrängt und selbst bindet. Dadurch wird die Zellatmung herunterreguliert und als Resultat unter anderem die ATP-Produktion gesenkt.

In der Photobiologie trifft nun Licht auf Moleküle (sogenannte Chromophoren), was die Elektronen nun durch die Photonenenergie dazu bewegt auf ein höher energetisches Orbital zu springen. Die so gespeicherte Energie kann nun für Zellprozesse genutzt werden. Es wurde festgestellt, dass ein wichtiges Chromophor im Bereich der LLLT in der Atmungskette der Komplex IV, die Cytochrom-C-Oxidase ist, da demonstriert werden konnte, dass dieser Komplex das Lichtspektrum absorbieren kann, welches aus dem NIR-Bereich für biologische Prozesse verantwortlich ist (Karu 1989 a, Karu 1989 b, Chaieb et al. 2015).

Das bei der LLLT eintreffende Licht trennt nun das Stickoxid von der CCO (Cytochrom-COxidase) und gibt so dem Sauerstoff die Möglichkeit gebunden zu bleiben. In der Konsequenz wird die Zellatmung hochreguliert und die ATP-Produktion und damit die Verfügbarkeit von Energie für die Zelle und ihrer Prozesse erhöht (Karu 1989 a). Gemessen werden konnte dieser Effekt unter anderem durch vermehrte ATP-Produktion der Zelle (Karu et al. 1995) und erhöht en Elektronentransport (Pastore et al. 1994). 


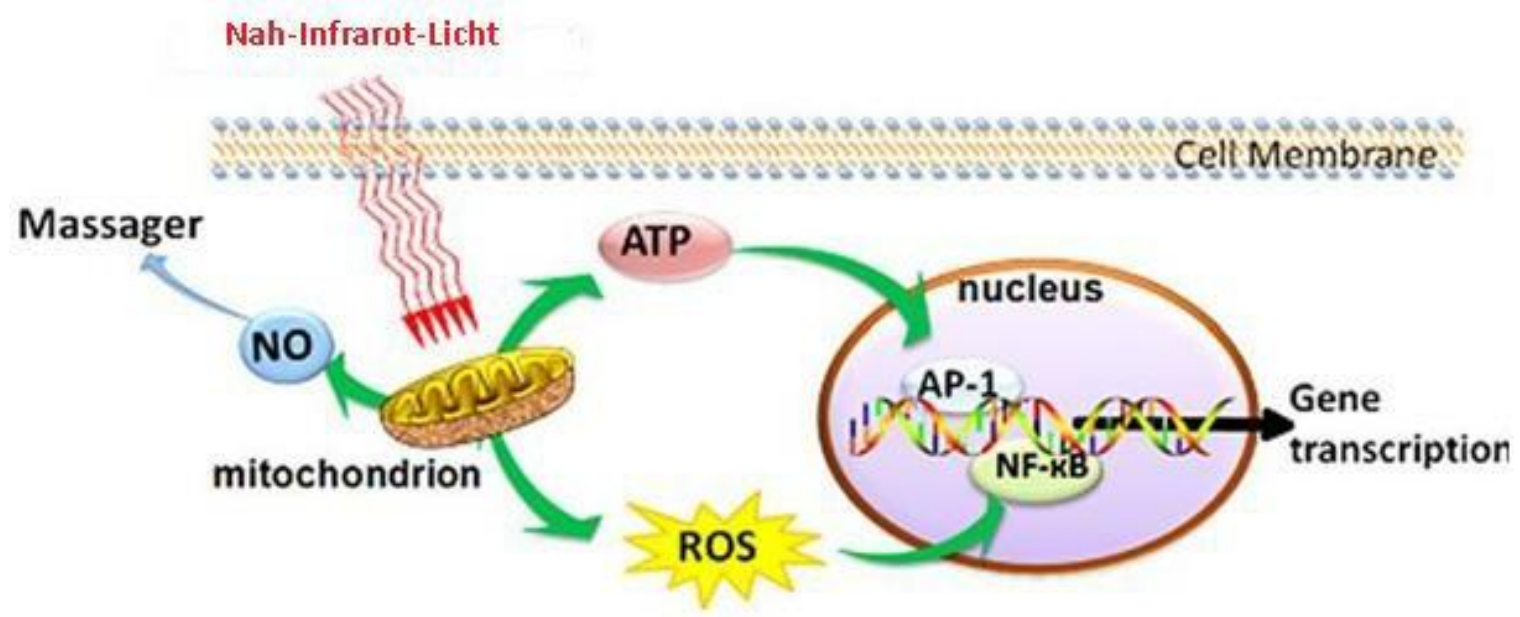

Abb. 1: „zelluläre Mechanismen der LLLT“ (modifiziert nach Chung et al. 2012, die Verwendung erfolgt mit freundlicher Genehmigung des Springer-Verlags): Nah-Infrarot-Licht wird durch Photoakzeptoren in den Mitochondrien absorbiert und führt zur Hochregulation der ATP-Produktion in der Atmungskette, was zu Aktivierung von Transkriptionsfaktoren führt.

Die Effektivität der LLLT als Therapieoption kognitiver Defizite nach Schlaganfall (Naeser 1997; Oron et al. 2006) und nach Fällen traumatischer Hirnverletzungen (Oron et al. 2012) konnte in Studien gezeigt werden. Außerdem wurde Potential für die Behandlung des Morbus Alzheimer durch LLLT gefunden (Sommer et al. 2012).

\subsection{Infrarotlaserstimulation}

„Near - infrared - low - level - Laser (NILLL) - Bestrahlung durchdringt Kopfhaut und Schädel und kann oberflächliche Schichten des zerebralen Kortex erreichen“ (Konstantinović et al 2013).

An der Universität Paderborn, Deutschland, wurde durch Dr. Detlef Schikora erstmals ein System von Laser-Akupunkturnadeln entwickelt, bichromatische Lasernadeln mit 685nm 
und 785nm, erste klinische Versuche wurden durch Dr. Michael Weber in Lauenförde, Deutschland, unternommen.

Transkranielle Infrarotlaserstimulation ist eine neue Möglichkeit der Hirnstimulation. Sie bietet die Möglichkeit, neuroplastische Veränderungen hervorzurufen mit hoher räumlicher Auflösung und einer tiefen Gewebepenetration (Weber et al. 2007).

Mit wissenschaftlichen Versuchen und ersten Veröffentlichungen zu diesem Thema wurde daraufhin an der Universität in Graz, Österreich, begonnen (Litscher et al. 2013). Laser-Akupunkturnadeln wirken äquivalent zu Metallnadeln, wobei die Lasernadeln hier Endstücke von Lichtleitfasern sind, welche den Strahl, der von den Laserdioden erzeugt wird, zur Körperoberfläche transportieren (Weber 2005). Entscheidend für den Erfolg hierbei ist die kleine Fläche der Nadeln, aus welcher das Laserlicht austritt. Die im Verhältnis zur Leistung gemessene kleine Fläche führt zu einer sehr großen Leistungsdichte und einer damit erhöhten Energieleistung, die Leistung steigt exponentiell mit Abnahme der Austrittsfläche an (Weber 2005). Dennoch ist das Risiko thermischer Nebenwirkungen sehr gering, da dieses erst in Leistungsbereichen weit oberhalb der vom Laser erreichten Leistungsdichte zu erwarten ist (Weber 2005). Dieses Laser-Akupunkturnadelsystem ermöglicht so also eine nichtinvasive Möglichkeit der simultanen Stimulation verschiedener Punkte, individuell kombinierbar (Litscher et al. 2013).

Dabei ist eine hohe Leistungsdichte bei einem Laser kleinerer Leistung, durch die kleine Applikatorfläche und den direkten Hautkontakt möglich und damit ein Einsatz in der Praxis. Erste Erfolge in klinischen Experimenten mit Laser-Akupunkturnadeln konnten beispielsweise bereits durch die Forschungsgruppe von Gerhard Litscher gezeigt werden, die in einer Zusammenarbeit mit dem Beijing-Krankenhaus für traditionelle 
chinesische Medizin die Erfolge manueller Nadel-Akupunktur und Laser-Akupunktur in der komplementären Behandlung von Burnout-Patienten verglichen. Hierbei teilten sie

ein Patientenkollektiv in zwei Gruppen, wobei eine Gruppe eine herkömmliche Akupunktur-Therapie erhielt, die andere Gruppe Akupunktur mit Lasernadeln. Dabei konnte anhand eines für die Studie neu entwickelten Scores gezeigt werden, dass LaserAkupunktur stärkere Erfolge auf das neurovegetative System hat als herkömmliche Akupunktur (Litscher et al. 2013).

\subsection{Transkranielle Magnetstimulation (TMS)}

Als Messverfahren wurde in dieser Studie das Stimulationsverfahren der transkraniellen Magnetstimulation (TMS) verwendet. Die Mechanismen sind dabei vom selben Prinzip wie bei der konventionellen Elektrostimulation, allerdings ist TMS sowohl nichtinvasiv als auch schmerzfrei, was seit der Einführung dieses Verfahrens durch Barker et al. (1985) vielfältige neue Möglichkeiten für Untersuchungen eröffnete.

Das von außen angelegte zeitlich veränderliche Magnetfeld dient dabei zum Aufbau eines elektrischen Feldes im Inneren des Gehirns, nach dem Faraday"schen elektromagnetischen Induktionsgesetz. Dieses besagt, dass ein zeitlich veränderliches elektrisches Feld bei zeitlicher oder räumlicher Änderung der magnetischen Flussdichte entsteht. Dabei ist die Anstiegszeit des magnetischen Feldes so kurz, wie möglich, um ein möglichst hohes elektrisches Feld zu induzieren.

Eine Magnetspule induziert dabei im darunter liegenden Kortex einen parallel zum Strom in der Spule verlaufenden Stromfluss. Durch die anatomisch unterschiedlichen Strukturen 
unter der Schädelkalotte, von Hirnhäuten, über graue und weiße Substanz bis hin zum Liquor cerebrospinalis, gilt jedoch auch eine, je nach Struktur, unterschiedliche elektrische Leitfähigkeit. Bevorzugt werden nun solche Nervenzellen stimuliert, welche mit ihren Axonen parallel zu dem elektrischen Feld angeordnet sind, welches mittels Magnetfeld induziert wird (Amassian et al. 1987). Befinden sich die in diesem Kortexareal gelegenen Neurone im unerregten Zustand ist ihre Membran durch ein charakteristisches Ruhemembranpotential gekennzeichnet. Durch den nun von außen erzeugten elektrischen Gradienten depolarisiert die Membran bis zu einem Schwellenwert, an welchem sich die Öffnungswahrscheinlichkeit depolarisierender lonenkanäle der Neuronen drastisch erhöht. Durch den folgenden Ionenstrom wird die Membran weiter depolarisiert und in der Zelle ein Aktionspotential ausgelöst.

Am schnellsten leiten die Neurone des kortikospinalen Trakts, wenn nun deszendierende Erregungswellen bis zum spinalen Motoneuron geleitet werden, von wo über synaptische Verschaltung schließlich exzitatorische postsynaptische Potentiale zum peripheren Axon des Muskels geleitet werden (Amassian et al. 1987). Hier kann nun durch Oberflächenelektroden ein Muskelsummenpotential als motorisch evoziertes Potential (MEP) aufgezeichnet und so die Erregung des Muskels quantifiziert werden.

Das TMS-Verfahren kann sowohl selbst als Methode zur Modulation kortikaler Exzitabilität genutzt werden und je nach Stimulationsparametern hemmend oder anregend auf die Neuronenpopulation wirken (Hallett 2000) als auch, wie im vorliegenden Fall, zur Beobachtung induzierter Neuroplastizität durch andere Stimulationsverfahren des Gehirns (Nitsche und Paulus 2000) durch die Möglichkeit, das Leitvermögen zentraler motorischer Bahnen zu messen (Hallett 2000). Durch die aufgezeichneten MEPs kann so eine Aussage über Veränderungen der kortikalen 
Exzitabilität und Nacheffekte durch Infrarotlaserstimulation getroffen werden.

\subsection{Ziel der vorliegenden Studie}

Die Effekte der transkraniellen Laserstimulation wurden in diversen Studien untersucht. So konnte gezeigt werden, dass bei einer kurzzeitigen Stimulation mittels low-level-laser therapy eine Reduktion der kortikalen Erregbarkeit bewirkt werden kann (Konstantinović et al. 2013). So wurde auch kürzlich eine Studie in der Klinik für Klinische Neurophysiologie der Universität Göttingen durchgeführt („Neuroplastic effects of transcranial near-infrared stimulation (tNIRS) on the motor cortex", Chaeib et al. 2014), welche nach 10-minütiger Stimulation mit tNIRS eine Verminderung der kortikalen Exzitabilität aufweisen konnte.

Chaieb et al. postulierten als mögliche Ursache der hemmenden Wirkung, dass tNIRS möglicherweise die Wirkung inhibierender neuronaler Netzwerke erleichtere, bzw. deren Einfluss auf kortikospinale Motoneurone durch Erhöhung GABAerger Neurotransmitter erleichtere. Auch die Möglichkeit, glutamaterge Auswirkungen zu verringern, was letztendlich in einer verminderten Amplitude resultiere, wurde diskutiert (Chaieb et al 2015).

Allerdings sind optimale Stimulationsbedingungen zur Beeinflussung der kortikalen Exzitabilität noch nicht vollständig untersucht.

Das Ziel der hier vorliegenden Studie ist es nun, festzustellen, ob die Applikationsdauer einer Stimulation mit tNIRS einen Einfluss auf die Veränderlichkeit der kortikalen Exzitabilität hat und wie sich die Langzeitergebnisse der Amplituden der durch TMS ausgelösten MEPs mit unterschiedlicher Stimulationsdauer verändern. Zu diesem Zweck werden eine 5- und eine 20-minütige Stimulationsdauer, sowie eine 10-minütige Sham- 
Stimulation mit 90-minütigen MEP-Nachmessungen verglichen. Dies soll im Weiteren auch helfen, optimale Stimulationsbedingungen zu bestimmen, um gegebenenfalls einen späteren klinischen Einsatz der tNIRS zu ermöglichen. 


\section{Material und Methoden}

\subsection{Studienteilnehmer}

An der hier vorliegenden Studie, welche an der Universitätsmedizin Göttingen, Klinik für Klinische Neurophysiologie durchgeführt wurde, haben 14 Probanden (sieben weiblich) im Alter zwischen 19 und 29 Jahren teilgenommen. Die Probanden wurden über Aushänge in Universitätsgebäuden sowie über universitätsinterne Internetplattformen rekrutiert.

Die Studie wurde von der ortsansässigen Ethikkomission genehmigt (21/03/12), und bei der Durchführung wurden die ethischen Standards der Deklaration von Helsinki (1964, aktuelles Amendement 64th WMA General Assembly, Fortaleza, Brazil, October 2013) eingehalten.

Vor Beginn der experimentellen Studiendurchführung wurden die Teilnehmer mehrmals mündlich und schriftlich aufgeklärt über den Versuchsablauf, mögliche Risiken und die Möglichkeit, die Studie jederzeit ohne Angabe von Gründen, beenden zu können. Alle Probanden gaben ihr schriftliches Einverständnis vor Beginn der Studie. Für ihre Teilnahme erhielten alle Probanden eine Aufwandsentschädigung.

\subsection{Ausschlusskriterien}

Die Ausschlusskriterien zur Teilnahme an der Studie wurden anhand der allgemeinen Sicherheitsrichtlinien für transkranielle Stimulation (Anand und Hotson 2002, Pascual- 
Leone et al. 1993, Wassermann 1998, Wassermann et al. 1996, Barker 1999, Brandt et al. 1997, Nitsche et al. 2008) festgelegt und beinhalteten: Epilepsie, Migräne, intrakranielle Metallimplantate jeder Art, das Tragen eines Herzschrittmachers, regelmäßige Einnahme jedweder Medikamente (ausgenommen orale Kontrazeptiva), Schwangerschaft, sonstige neurologische und psychiatrische Erkrankungen sowie schwere und/oder chronische körperliche Erkrankungen sowie die Teilnahme an einer anderen Studie innerhalb der letzten sechs Wochen. Die teilnehmenden Probanden wurden vor Beginn der Studie ärztlich untersucht und erst nach Ausschluss oben genannter gesundheitlicher Einschränkungen vom Arzt zur Teilnahme an den Messungen zugelassen.

\subsection{Elektrophysiologische Messungen}

\subsubsection{Vorbereitung}

Während der Messungen saßen die Probanden in bequemer Sitzposition in einem Stuhl mit sowohl verstellbarer Rückenlehne als auch Kopfstütze. Die rechte obere Extremität wurde bequem gelagert, um eine maximale muskuläre Entspannung während der Messungen zu gewährleisten.

An die rechte Hand der Probanden wurden zwei $\mathrm{Ag} / \mathrm{AgCl}$ - Oberflächenelektroden (Durchmesser $9 \mathrm{~mm}$ ) festgeklebt, welche zur EMG-Ableitung der durch TMS induzierten MEPs dienten. Dabei wurde der elektrische Widerstand zwischen Haut und Elektrode mit Elektrodengel verringert, und es wurde darauf geachtet, die Elektroden so fest zu kleben, dass sie sich durch Bewegung oder Schwitzen nicht lösen konnten. Die differente 
Elektrode wurde über der Mitte des Muskelbauchs des M. interosseus dorsalis I (FDI) als Zielmuskel, die indifferente Elektrode am entsprechenden Muskelsehnenansatz des FDI, an der Grundphalanx des zweiten Fingers, befestigt. Eine Erdungselektrode wurde zwischen den ableitenden Elektroden und dem Ort der Stimulation, am Handgelenk, platziert.

Mit einem im Ableitegerät integrierten Tiefpassfilter (2 kHz), sowie einem aufgrund der diversen durch Wechselstrom verursachten Störsignale in der Laborumgebung zwischengeschalteten Notch-Filter $(50 \mathrm{~Hz})$ wurde das vorverstärkte Rohsignal in einen Frequenzbereich eingegrenzt.

Die Signaldaten wurden digitalisiert mittels eines Micro 1401 AD Converters (Cambridge Electronic Design, Cambridge, UK) und mit Hilfe von Signal Software (Cambridge Electronic Design, Version 2.13) kontrolliert. Daraufhin wurden die Daten auf einer Festplatte eines persönlichen Computers zur späteren Analyse gespeichert.

\subsubsection{TMS-Messungen}

In den durchgeführten experimentellen Untersuchungen wurden mit einem Magstim 200 Magnetstimulator (Magstim Company, Whiteland, Wales, UK) monophasische Magnetimpulse erzeugt. Die verwendete achtförmige Doppelspule (Durchmesser einer Spule $=70 \mathrm{~mm}$, Spitze magnetisches Feld = 2,2 Tesla, Magstim Company, Whiteland, Wales, UK) wurde orientierend über dem primären motorischen Handareal des linken Kortex positioniert. Dabei wurde von einer ungefähren Position dieses Areals 4-5 cm lateral und 1-2 $\mathrm{cm}$ anterior des Vertex ausgegangen. 
Um die technische Stromrichtung posterior-anterior verlaufen zu lassen, zeigte der Spulengriff nach postero-lateral, möglichst im $45^{\circ}$ - Winkel zur Sagittallinie. Der optimale Stimulationsort (engl. motor hot spot), das heißt der Ort größter Dichte kortikospinaler Fasern zum Zielmuskel und damit der Ort, an dem mittels TMS eine maximale motorische Antwort im Zielmuskel hervorgerufen wird, wurde ermittelt. Zu diesem Zweck wurden Einzel-Puls- Testreize über der M1-Region der linken Hemisphäre mittels der Spule generiert und die Spule dabei langsam von anterior nach posterior entlang der Sagittallinie bewegt.

Nach Auffinden des optimalen Stimulationsorts wurde dieser farblich gekennzeichnet, um inn wiederauffinden und während der gesamten Untersuchung beibehalten zu können. Während jeder folgenden Messung der Untersuchung wurde dort die Doppelspule mit den Händen durch den Untersucher fixiert.

\subsubsection{Schwellenwertbestimmung}

Zunächst wurde an dem ermittelten optimalen Stimulationsort die kortikale motorische Reizschwelle (engl. resting motor threshold, RMT) bestimmt. Dazu wurde mit einem überschwelligen Testreiz stimuliert, dessen Intensität dann stufenweise um 1\% reduziert wurde, bis die Intensität erreicht war, die bei drei von fünf Stimulationsversuchen ein MEP hervorrief, dessen Amplitude über $50 \mu \mathrm{V}$ von Spitze zu Spitze überstieg. Diese Stimulationsintensität wurde als RMT-Wert festgelegt. Daraufhin erfolgte die Erhebung des Werts der aktiven motorischen Schwelle (engl. active motor threshold, AMT). Da bekannt ist, dass eine willkürliche Kontraktion des Zielmuskels durch die Faszilitation

nicht nur die Amplitude, sondern auch die Latenz des MEP beeinflusst (Ellaway et al. 
1998; Kiers et al. 1993; Mills et al. 1987), wobei minimale MEP-Latenzen bereits ab 10\% der maximalen Willküraktivität des Zielmuskels erreicht werden (Mills et al. 1987; Ravnborg und Dahl 1991), wurden die Probanden für die Bestimmung der AMT gebeten, eine Pflasterrolle zwischen Daumen und Zeigefinger zu nehmen. Diese Rolle sollte mit etwa $25 \%$ iger-Kraft komprimiert und dadurch eine tonische Kontraktion des Zielmuskels (FDI) erreicht werden. Die Intensität der Stimulation wurde unter TMS-Impulsgabe weiter schrittweise reduziert, bis die minimale Intensität erreicht wurde, mit der in mindestens drei aus sechs Impulsen noch ein MEP ausgelöst werden konnte, dessen Amplitude die maximale muskuläre Hintergrundaktivität um $15 \%$ überstieg Nach dieser Ermittlung der Schwellenwerte folgte nun das Aufzeichnen der Baseline. Zu diesem Zweck wurde eine Intensität des Magnetstimulators gewählt, die eine MEPAmplitude erzeugte, deren Peak-to-Peak-Wert bei durchschnittlich $1 \mathrm{mV}$ lag. Nach einer Aufzeichnung von 30 MEPs mit einer Frequenz von $0,25 \mathrm{~Hz}$ mit Hilfe des Computerprogramms ,Signal" wurde das Erreichen eines Mittelwerts der Amplituden von $1 \mathrm{mV}$ mittels der Software ,NuCursor" vom Untersucher überprüft.

\subsection{Stimulationstechniken}

\subsection{1. tNIRS}

Nach erfolgter Schwellenwertbestimmung und Baseline-Aufzeichung fand nun die Stimulation mittels Infrarotlaser statt.

Zum Zeitpunkt der Studie waren noch keine ausreichend geprüften einheitlichen Protokolle für exakte Stimulationsbedingungen, wie beispielsweise Dauer, Intensität und Stärke vorhanden. Zur besseren Vergleichbarkeit mit anderen Studien wurden deshalb 
die aus unseren Labormessungen bekannten Stimulationsbedingungen einer vorangegangen Studie der Abteilung (Chaieb et al. 2015) übernommen.

Für die Stimulation wurden vier Laser-Dioden, befestigt an perkutanen Akupunkturnadeln über dem ermittelten ,hot-spot' des Kortex angebracht. Dabei wurden sie mit Hilfe eines wie eine Krone um den Kopf des Probanden gelegten gepolsterten Rahmens und daran befestigten Drahthalterungen während der Stimulation an diesem Ort gehalten. Der Rahmen konnte dabei individuell dem Kopf des Probanden für optimalen Halt angepasst werden.

Die perkutanen Laser Akupunkturnadeln bestehen aus rostfreiem Edelstahl, sind wieder verwertbar und wurden nach jedem Gebrauch sorgfältig gereinigt und desinfiziert. Sie sind mit einem optischen Fiberkabel verbunden und erreichen eine Leistung von $50 \mathrm{~mW}$ pro Diode bei einer Wellenlänge von $810 \mathrm{~nm}$. Durch die flexiblen optischen Fiberkabel wird ein minimaler Verlust der Energie an die Lasernadel sichergestellt bei Leitung des Lichts, was eine hohe optische Dichte am Ende der Lasernadel gewährleistet. 


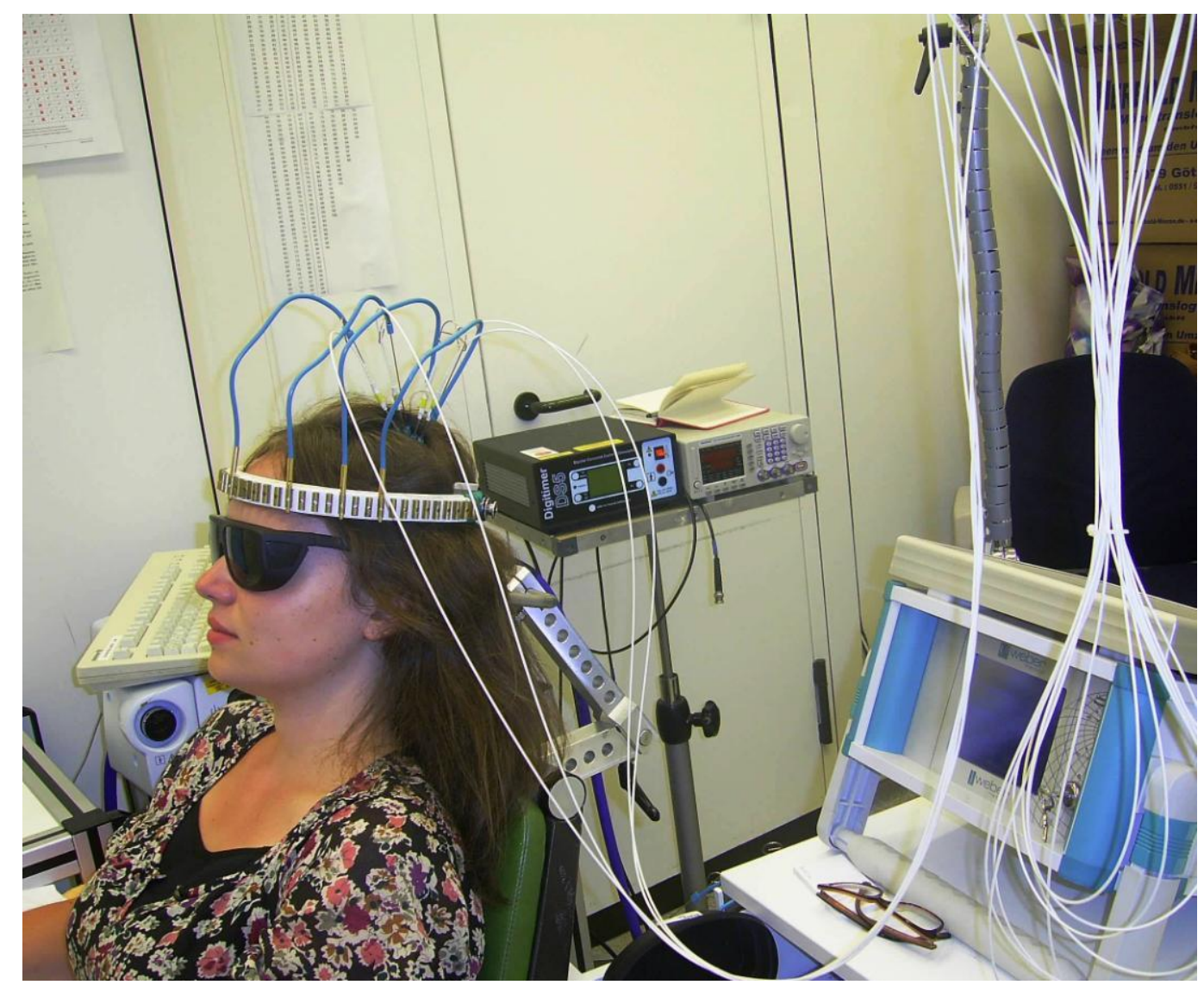

Abb. 3 Versuchsaufbau mit perkutanen Laserakupunkturnadeln, angeschlossen am Laser-Stimulator (WeberMedical, GmbH, Deutschland), befestigt über eine Krone am Kopf der Probandin

Der Laser-Stimulator (WeberMedical, GmbH, Deutschland) wurde vom Untersucher vor jeder Stimulation für die jeweilige Sitzung programmiert auf eine Leistung von $35 \%$ und eine je nach Sitzung variierende Stimulationsdauer von 5 oder 20 Minuten. Die Leistung der Lasernadeln wurde dabei so eingestellt, dass die Probanden keinen Unterschied bei Aktivität oder Inaktivität der Lasernadel spürten. Nach geschehener Stimulation stoppte das Programm automatisch. Zum Schutz der Retina vor Laserstrahlen trugen alle im Raum anwesenden Personen Laserschutzbrillen, auch während der Plazebo-Stimulation.

Für die Plazebo-Stimulation wurde der Stimulator nur programmiert, um den Probanden keinen Situationsunterschied zur Stimulationssitzung spüren zu lassen, aber nicht gestartet. 
Als Einfachblindstudie war für den Probanden nicht ersichtlich, welche der genannten Stimulationen die Plazebo-Stimulation darstellte.

Nach Ablauf der jeweiligen Stimulationszeit, erfolgte sofort die erste Nachmessung zur Ermittlung der Nacheffekte der Laserstimulation mit Hilfe der TMS mit $0,25 \mathrm{~Hz}$ bei 30 Impulsen. Es wurde dabei dieselbe Intensität verwendet, die vor der Stimulation für die Baseline ermittelt und verwendet worden war.

Weitere Messungen der Nacheffekte folgten jeweils nach fünf, zehn, 15, 20, 25, 30, 40, 50, 60 und 90 Minuten nach Stimulationsende. Zwischen den einzelnen Sitzungen wurde ein Mindestabstand von vier Tagen eingehalten, zum Ausschluss gegebenenfalls auftretender Nacheffekte der vorhergehenden Sitzung auf die Nachfolgende.

\subsection{Statistik}

Die erzeugten MEPs wurden zunächst jeweils einzeln ausgewertet. MEPs mit EMG Artefakten wurden gelöscht. Durch die Software "Signal" waren Kurven dargestellt worden, welche durch das NuCursor-Programm weiter analysiert wurden. Hierbei wurde die Amplitude der MEPs (jeweilige Differenz zwischen oberem und unterem Umschlagpunkt, "peak-to-peak") automatisch durch das NuCursor-Programm (IoN, UCL, London, UK) berechnet. Der Mittelwert wurde für jeden Zeitpunkt bestimmt. So wurden die Mittelwerte der Amplituden der MEPs für jeden Zeitpunkt der Baseline, also vor Stimulation, sowie für die Zeitpunkte $0,5,10,15,20,25,30,40,50,60$ und 90 Minuten nach der Stimulation berechnet.

Zur Baseline genormte MEPs wurden daraufhin mittels univarianter Varianzanalyse 
(ANOVA) analysiert. Dabei wurde mit ANOVA die Effektivität der Stimulation gegenüber Plazebo untersucht.

Es wurden drei Hauptbedingungen (5 minütige tNIRS, 20 minütige tNIRS und PlazeboMessung) untersucht. ANOVA wurde mit den Faktoren "Stimulationsbedingung" (tNIRS vs. Plazebo), "Stimulationsdauer" (fünf Minuten vs. 20 Minuten) und „Zeitpunkt”, an welchem die Nachmessung erfolgte (11 Ebenen: 0, 5, 10, 15, 20, 25, 30, 40, 50, 60, 90 Minuten nach Stimulationsende): (Stimulationsdauer (tNIRS vs. Plazebo) x Zeit (0, 5, 10, $15 ; 20,25 ; 30,40,50,60,90$ Minuten post-Stimulation) jeweils als repeated measures gerechnet.

Stimulationsdauer, -bedingung und Zeitpunkt waren hierbei unabhängige Variablen, die Höhe der MEP - Amplituden die abhängige Variable. Effekte wurden bei $p \leq 0.05$ als signifikant betrachtet. Im Falle eines signifikanten Effekts wurde ein t-Test durchgeführt. Um die Werte der MEPs innerhalb der Gruppe zwischen Baseline und Post-StimulationsMessungen zu vergleichen wurde ein t-Test durchgeführt.

Alle nachfolgenden Daten sind als Mittelwert und Standardfehler des Mittelwerts (SEM) angegeben. 


\section{Ergebnisse}

Nachfolgend werden die Ergebnisse der durchgeführten Studie dargestellt.

\subsection{Ziel}

Ziel war die Feststellung einer Veränderlichkeit der kortikalen Exzitabilität durch unterschiedliche Applikationsdauer bei einer Stimulation mit tNIRS. Dazu wurden nach 5und 20-minütiger Stimulationsdauer sowie einer Plazebomessung über 90 Minuten die Amplituden der durch TMS ausgelösten MEPs gemessen. Die gemessenen Amplituden der MEPs nach der Stimulation wurden verglichen mit einer Baseline von MEP-Amplituden, die vor der Stimulation gemessen wurden sowie mit den MEP-Amplituden der PlazeboMessung.

Alle teilnehmenden Probanden tolerierten die Stimulation sehr gut. Keine der experimentellen Sitzungen musste aufgrund von Nebenwirkungen unter- oder abgebrochen werden.

RMT-Werte (Stimulationsintensität), AMT-Werte (active motor threshold) und BaselineMEP- Werte wurden verglichen zwischen tNIRS und Plazebo-Bedingungen, hierbei wurde der t-Test verwendet. Dabei konnte kein signifikanter Unterschied zwischen den Messungen festgestellt werden (alle $p>0.05$ ).

\subsection{Deskriptive Statistik}

Die durchschnittlichen Mittelwerte der MEP-Amplituden, sowie die Standardabweichung der einzelnen Messungen nach 5 minütiger Stimulation, 10 minütiger Stimulation und die 
Plazebo - Stimulation zeigt die nachfolgende Tabelle (Tab. 1).

\begin{tabular}{|l|l|l|l|l|l|l|}
\hline & \multicolumn{2}{|c|}{ Plazebo } & \multicolumn{2}{c|}{ 5 Min tNIRS } & \multicolumn{2}{c|}{ 20 Min tNIRS } \\
& MW & \multicolumn{2}{|c|}{ MW } & MW & SD \\
\hline Baseline & 0,99 & 0,99 & 1,06 & 0,13 & 1,00 & 0,11 \\
\hline 0 Min & 1,04 & 1,04 & 0,92 & 0,33 & 1,10 & 0,15 \\
\hline 5 Min & 1,00 & 1,00 & 1,02 & 0,31 & 1,23 & 0,29 \\
\hline 10 Min & 1,01 & 1,01 & 0,95 & 0,33 & 1,21 & 0,21 \\
\hline 15 Min & 1,04 & 1,04 & 1,00 & 0,40 & 1,17 & 0,20 \\
\hline 20 Min & 1,06 & 1,06 & 0,93 & 0,24 & 1,11 & 0,32 \\
\hline 25 Min & 1,01 & 1,01 & 0,85 & 0,27 & 1,10 & 0,28 \\
\hline 30 Min & 0,98 & 0,98 & 1,03 & 0,38 & 1,11 & 0,20 \\
\hline 40 Min & 1,08 & 1,08 & 1,09 & 0,54 & 0,92 & 0,21 \\
\hline 50 Min & 0,96 & 0,96 & 0,98 & 0,31 & 1,00 & 0,33 \\
\hline 60 Min & 1,04 & 1,04 & 1,00 & 0,27 & 1,00 & 0,31 \\
\hline 90 Min & 1,05 & 1,05 & 0,99 & 0,26 & 1,13 & 0,31 \\
\hline
\end{tabular}

Tab. 1: alle Werte sind in $\mathrm{mV}$ angegeben und auf zwei Nachkommastellen gerundet. $\mathrm{MW}=$ Mittelwert, SD = Standardabweichung, $5 \mathrm{Min}$ tNIRS = 5-minütige transkranielle Nahinfrarot Stimulation, 20 Min tNIRS = 20-minütige transkranielle Nahinfrarot Stimulation

\subsection{Varianzanalysen}

\subsubsection{Vergleich Stimulation (5 minütige tNIRS und 20 minütige $t N I R S)$ und Plazebostimulation}

Durch ANOVAs mit repeated measurements konnte ein signifikanter Haupteffekt für den

Faktor "Stimulationsbedingung" $(F(2,28)=2,67, p=0,084)$ nachgewiesen werden (siehe Tab. 2).

Der Faktor „Zeitpunkt" ergab keine Signifikanz: $F(10,140)=1,169, p=0,32$. Eine Interaktion zwischen den Faktoren "Stimulationsbedingung" und „Zeitpunkt" bestand nicht $(F(20,280)=1,29, p=0,18$. 


\begin{tabular}{|l|c|c|c|}
\hline \multicolumn{1}{|c|}{ Faktor } & df & F & p \\
\hline $\begin{array}{l}\text { Stimulationsbedingung } \\
\text { (tNIRS vs. Plazebo) }\end{array}$ & 2,28 & 2,67 & 0,084 \\
\hline Zeitpunkt & 10,140 & 1,169 & 0,32 \\
\hline Interaktionen & 20,280 & 1,29 & 0,18 \\
\hline
\end{tabular}

Tab. 2: Stimulationsbedingung: tNIRS vs. Plazebo

\subsubsection{Effekte 5-minütiger tNIRS-Stimulation}

Nach 5-minütiger tNIRS-Stimulation zeigte die Messung der durch TMS ausgelösten Amplituden der MEPs eine Reduktion der kortikalen Exzitabilität um 10-20 \% (Abbildung 4).

Der Student,s t-Test wurde zum Vergleich der MEP-Amplituden nach Stimulationsende $(0,5,10,15,20,25,30,40,50,60,90$ Min) mit den MEP-Amplituden der Baseline durchgeführt. Die Analyse mit dem Student"s t-Test zeigte signifikant gesunkene MEPs innerhalb der 25 mit der Baseline verglichenen MEPs $(p<0.05)$.

Nach ANOVAs mit Messwiederholungen wurde der Haupteffekt verglichen zur PlazeboStimulation mit der Variablen „Stimulationsdauer" jedoch nicht signifikant $(F(1,14)=1.95$, $\mathrm{p}=0.18)$. 


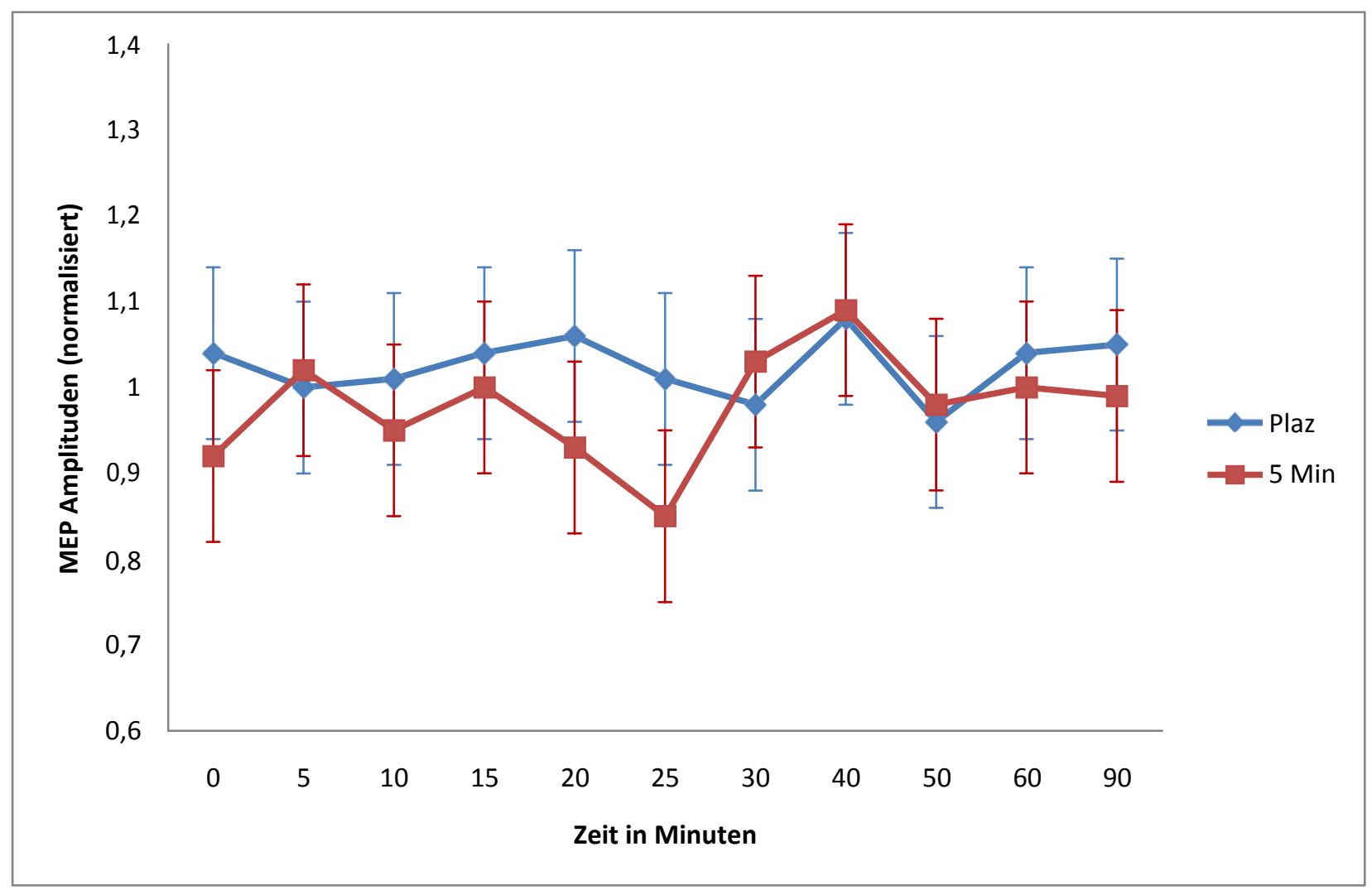

Abb. 4: Plazebo-Stimulation und 5minütige tNIRS im Vergleich

Grafische Darstellung der MEP-Amplituden nach 5-minütiger tNIRS-Stimulation. Daten gemittelt aus je 14 Einzelmessungen pro Messzeitpunkt an 14 Probanden. Die senkrechten Balken stellen den Standardfehler dar.

Der Haupteffekt der Variablen „Zeitpunkt“ $(F(10,140)=0.88, p=0.56)$ war nicht signifikant. Die Variablen „Stimulationsdauer" und „Zeitpunkt" zeigten keine signifikante Interaktion $(F(10,110)=0.51, p=0.88$, siehe Tab. 3).

\begin{tabular}{|l|c|c|c|}
\hline \multicolumn{1}{|c|}{ Faktor } & df & F & p \\
\hline $\begin{array}{l}\text { Stimulationsdauer } \\
\text { (5 Min. vs. Plazebo) }\end{array}$ & 1,14 & 1,95 & 0,18 \\
\hline Zeitpunkt & 10,140 & 0,88 & 0,56 \\
\hline Interaktionen & 10,110 & 0,51 & 0,88 \\
\hline
\end{tabular}

Tab. 3: Stimulationsdauer: 5 Minuten tNIRS vs. Plazebo 


\subsubsection{Effekte 20-minütiger tNIRS-Stimulation}

Nach 20-minütiger tNIRS-Stimulation zeigte die Messung der durch TMS ausgelösten Amplituden der MEPs eine Steigerung der kortikalen Exzitabilität um 20-30\% (siehe Abbildung 5).

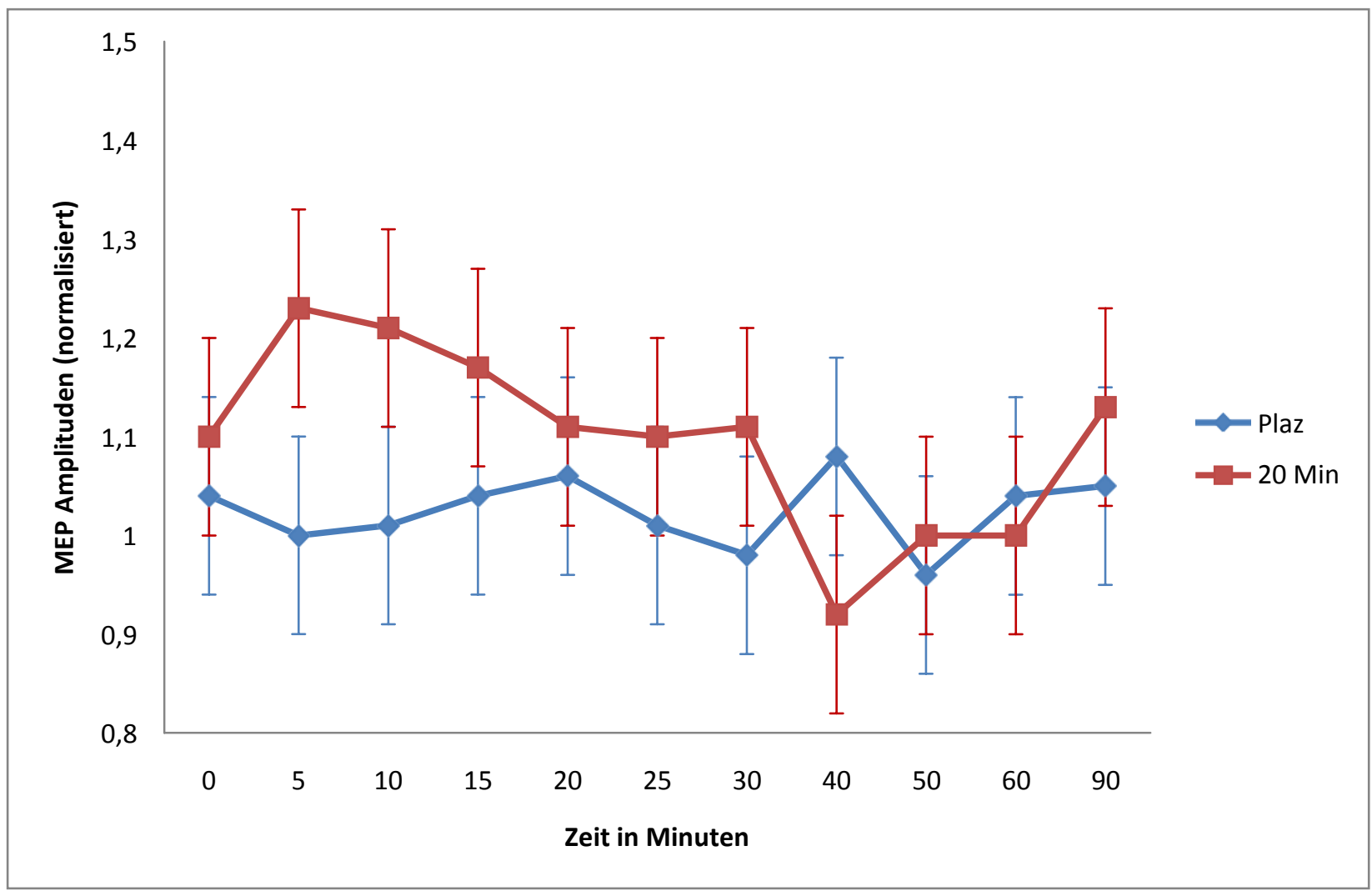

Abb. 5: Plazebo-Stimulation und 20 minütige tNIRS im Vergleich Grafische Darstellung der MEP-Amplituden nach 20-minütiger tNIRS-Stimulation. Daten gemittelt aus je 14 Einzelmessungen pro Messzeitpunkt an 14 Probanden. Die senkrechten Balken stellen die Standardabweichung dar.

Der Student"s t-Test wurde zum Vergleich der MEP - Amplituden nach Stimulationsende $(0,5,10,15,20,25,30,40,50,60,90$ Minuten) mit den MEP-Amplituden der Baseline durchgeführt. Der Test zeigte signifikant angestiegene MEPs 5, 15 und 30 Minuten nach Stimulation im Vergleich zur Baseline $(\mathrm{p}<0.05)$. 
Verglichen mit der Plazebo-Stimulation, zeigten jedoch wiederholte Messungen von ANOVA keinen signifikanten Effekt der Variablen "Stimulationsdauer" $(F(1,14)=1.21$, $\mathrm{p}=0.29)$. Der Haupteffekt der Variablen „Zeitpunkt“ $(F(10,140)=1.61, p=0.11)$ und die Interaktion zwischen "Stimulationsdauer“ und „Zeitpunkt“ waren hingegen nicht signifikant $(F(10,110)=1.48, p=0.15$, siehe Tab. 4).

\begin{tabular}{|l|c|c|c|}
\hline \multicolumn{1}{|c|}{ Faktor } & df & F & p \\
\hline $\begin{array}{l}\text { Stimulationsdauer } \\
\text { (20 Min. vs. Plazebo) }\end{array}$ & 1,14 & 1,21 & 0,29 \\
\hline Zeitpunkt & 10,140 & 1,61 & 0,11 \\
\hline Interaktionen & 10,110 & 1,48 & 0,15 \\
\hline
\end{tabular}

Tab. 4: Stimulationsdauer: 20 Minuten tNIRS vs. Plazebo

\subsubsection{Vergleich 5 - minütige und 20 - minütige Stimulation}

Vergleicht man die 5 - Minuten - und 20 - Minuten - Messungen, zeigen die Werte nach ANOVAs mit Messwiederholungen berechnet einen Haupteffekt „Stimulationsdauer“, der das Signifikanznivea knapp verfehlt $(F(1,14)=2.52, p=0.06)$. Der Haupteffekt der Variablen „Zeitpunkt“ war nicht signifikant $(F(10,140)=1.23, p=0.27)$.

Die Variablen „Stimulationsdauer" und „Zeitpunkt“ wiesen jedoch eine knapp signifikante Interaktion auf $(F(10,110)=1.89, p=0.05$, siehe Tab. 5 und Abbildung 6).

Mittels des daraufhin durchgeführten Student,,s t-Tests konnte ein signifikanter Unterschied zwischen den beiden Bedingungen bei 0 Minuten $(p=0,009)$, bei 5 Minuten $(p=0,024)$ und bei 25 Minuten $(p=0,01)$ festgestellt werden. Knapp verfehlt wurde das Signifikanzniveau bei 10 Minuten $(p=0,06)$. 


\begin{tabular}{|l|c|c|c|}
\hline \multicolumn{1}{|c|}{ Faktor } & df & F & p \\
\hline $\begin{array}{l}\text { Stimulationsdauer } \\
\text { (5 Min. vs. 20 Min.) }\end{array}$ & 1,14 & 2,52 & 0,06 \\
\hline Zeitpunkt & 10,140 & 1,23 & 0,27 \\
\hline Interaktionen & 10,110 & 1,89 & 0,05 \\
\hline
\end{tabular}

Tab. 5: Stimulationsdauer: Vergleich 5 Minuten tNIRS vs. 20 Minuten tNIRS

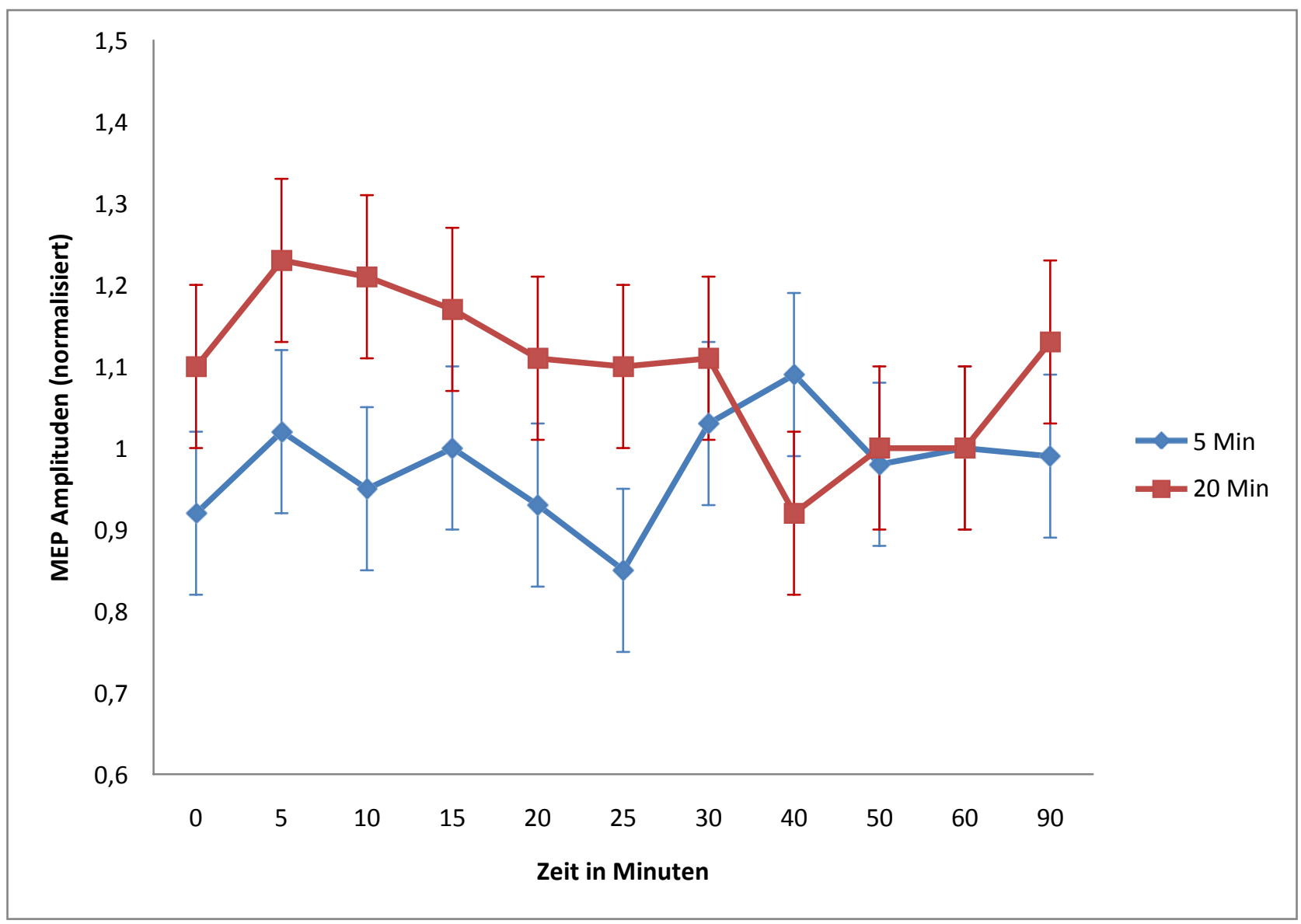

Abb. 6: 5-minütige tNIRS und 20-minütige tNIRS im Vergleich

Grafische Darstellung der MEP-Amplituden nach 5- und 20-minütiger tNIRS. Daten gemittelt aus je 14 Einzelmessungen pro Messzeitpunkt an 14 Probanden. Die senkrechten Balken stellen die Standardabweichung dar. 


\section{Diskussion}

\subsection{Ziel der Studie und Diskussion der Ergebnisse}

In vorhergehenden Studien konnte bei Einsatz von tNIRS bereits gezeigt werden, dass damit eine Möglichkeit der Modulierbarkeit der kortikalen Exzitabilität besteht (Konstantinović et al. 2013, Chaieb et al. 2015). Beispielsweise zeigten Chaieb et al. (2015), dass eine 10-minütige Stimulationsdauer mit tNIRS die kortikale Exzitabilität signifikant hemmt. Bisher sind allerdings die Stimulationsbedingungen, wie beispielsweise die Dauer der Stimulation, noch weitgehend ungeklärt. In der vorliegenden Studie wurde dies deshalb näher untersucht, auch um möglichst einheitliche gut untersuchte Stimulationsprotokolle für bessere Vergleichbarkeit innerhalb verschiedener Studien zu erstellen. Ziel war die Festlegung der nötigen Stimulationsdauer, die die kortikale Exzitabilität verändert. Gemessen wurde dies anhand der Änderung der MEP-Amplitudenhöhe nach Stimulation mit tNIRS unterschiedlicher Dauer im Vergleich zur MEP-Amplitudenhöhe der Baseline.

\subsubsection{Ergebnisse der hier vorliegenden Studie}

Als erstes betrachteten wir den Effekt von tNIRS im Vergleich zur Plazebostimulation, um zu bestätigen, dass generell ein Effekt der Stimulation besteht. Es lässt sich auch in den hier vorliegenden Daten bestätigen, dass tNIRS eine signifikante Veränderung der MEPAmplitudenhöhe und damit der kortikalen Exzitabilität hervorruft im Vergleich zur Plazebostimulation (siehe 3.3.1.) Betrachtet man nun im Einzelnen die Effekte 
unterschiedlicher Dauer der Stimulation, so lassen sich nachfolgende Beobachtungen machen.

Nach 5-minütiger tNIRS konnte anhand der verglichenen MEP-Amplitudenhöhen zur Baseline und zur Plazebo-Messung eine Reduktion festgestellt werden, allerdings nur um 10- 20\%, besonders zum Zeitpunkt 25 Minuten nach Stimulationsende.

In der Grafik (siehe Abb. 4) wird besonders deutlich, dass während der ersten 15 Minuten nach Stimulation keine Veränderung der MEP-Amplitudenhöhe auftritt. Es scheint also eine Art Latenzphase über die ersten 15 Minuten zu bestehen, bis der Effekt der tNIRS sichtbar wird. Die könnte die Theorie einer neuronalen Modulation bestätigen, die erst stattfinden muss und Zeit benötigt, weshalb die kortikale Exzitabilität deshalb leicht zeitverzögert und nicht unmittelbar verändert ist.

Weiterhin kann man deutlich sehen, dass ab dem Zeitpunkt 15 Minuten nach der Stimulation über einen Zeitraum von 10 Minuten andauernd die MEP-Amplitudenhöhe kontinuierlich abnimmt, auch im Vergleich zur Plazebomessung betrachtet. In dieser bleibt die MEP-Amplitudenhöhe annähernd unverändert. Zum Zeitpunkt 25 Minuten post stimulationem hat die gemessene Amplitudenhöhe ihren Tiefpunkt erreicht. 


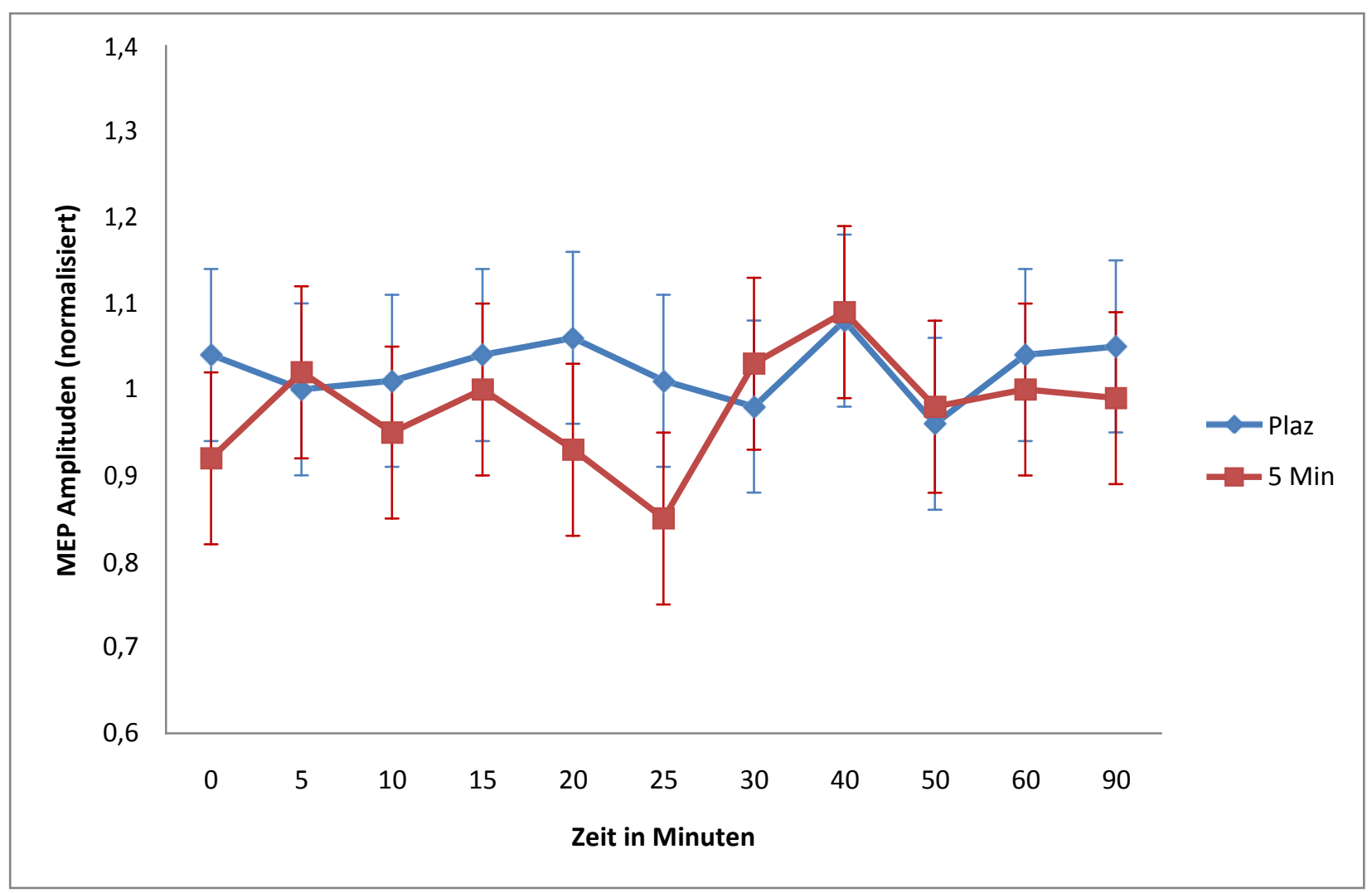

Abb. 4: Plazebo-Stimulation und 5minütige tNIRS im Vergleich

Grafische Darstellung der MEP-Amplituden nach 5-minütiger tNIRS-Stimulation. Daten gemittelt aus je 14 Einzelmessungen pro Messzeitpunkt an 14 Probanden. Die senkrechten Balken stellen den Standardfehler dar.

Zwar verfehlte der Faktor "Stimulationsdauer" insgesamt in ANOVAs berechnet das Signifikanzniveau, dennoch ist eine nicht signifikante Senkung der MEP-Amplitudenhöhe nach 5-minütiger tNIRS erkennbar. Ein Effekt von tNIRS scheint also zu bestehen und die oben erwähnten Ergebnisse der Studie von Chaieb et al. bestätigen zu können. Allerdings scheint die Stimulationsdauer von 5 Minuten nicht ausreichend zu sein, die kortikale Exzitabilität längerfristig zu modulieren. So kann kein signifikanter Effekt hervorgebracht werden. Die Amplitudenhöhe steigt nach 25 Minuten wieder an und weist keine signifikanten Veränderungen im Vergleich zur Plazebostimulation mehr auf. Es scheint sich also am ehesten um eine kurzfristige Modulation der kortikalen Exzitabilität zu handeln, deren Effekt zeitlich sehr begrenzt ist. Dieser zeitlich terminierte Effekt 
könnte unter Anderem auch in der Dauer der Stimulation begründet sein.

Nach 20-minütiger tNIRS hingegen zeigte die Messung der durch TMS ausgelösten Amplituden der MEPs eine Steigerung der kortikalen Exzitabilität um 20-30\%. Der $t$-Test zeigte signifikant angestiegene MEPs 5, 10, 15 und 30 Minuten nach Stimulation im Vergleich zur MEP-Amplitudenhöhe der Baseline.

Beim Messzeitpunkt 40 Minuten nach Stimulation fällt die Amplitudenhöhe allerdings stark ab, an den nachfolgenden Messzeitpunkten besteht kaum noch ein Unterschied zur gemessenen Amplitudenhöhe der Plazebostimulation.

Eine 20-minütige Stimulation führt also zur nachhaltigen Veränderung der MEPAmplitudenhöhe bis zu 30 Minuten nach Stimulation und ist in der Lage die kortikale Exzitabilität signifikant zu erhöhen. Man kann hieraus ableiten, dass eine längere Stimulationsdauer die kortikale Exzitabilität mehr beeinflusst, als kürzere Stimulationsdauer. Auf die Nachhaltigkeit der Veränderung scheint die Stimulationsdauer allerdings nur begrenzt Einfluss zu zeigen. So sieht man bei 5 -minütiger tNIRS einen Effekt bis 25 Minuten nach Stimulationsende, bei 20-minütiger Dauer bis 30 Minuten danach. Da auch Chaieb et al. mit ihrer Studie zur 10-minütigen Stimulation eine Nachwirkung des Effekts bis zu 30 Minuten beobachten konnten, scheint dies die bisher erreichbare Dauer einer Veränderung zu sein. Möglicherweise ist hier aber auch die immer konstant gehaltene Intensität der Stimulation ein limitierender Faktor und die Nachhaltigkeit wird nicht allein durch die Dauer der stattfindenden Stimulation beeinflusst.

Außerdem findet sich die interessante Beobachtung, dass mit der Dauer der Stimulation die Erregbarkeit der kortikalen Strukturen anzusteigen scheint, wohingegen bei kürzerer Dauer (5-minütige tNIRS und 10-minütige tNIRS, Chaieb et al. 2015) die Erregbarkeit 
nachweislich reduziert wurde. Die Dauer der Stimulation als alleiniger Faktor hat also nicht nur Einfluss auf die Stärke und Signifikanz der ausgelösten Effekte, sondern auch auf die Art, wie moduliert wird. Vergleicht man den Effekt 10-minütiger und 20-minütiger Stimulation so scheinen mit verdoppelter Stimulationsdauer sogar gegenteilige Effekte hervorgerufen zu werden. Dieser neue unerwartete zusätzliche Effekt des Faktors der Stimulationsdauer sollte in zukünftigen Überlegungen mit bedacht werden. Interessant wäre sicherlich auch die weitere Beobachtung noch längerer Stimulationsdauern, lässt sich doch nicht sicher ableiten, ob eher eine lineare Verstärkung der Exzitabilität oder im Gegenteil nach Erreichen eines Maximums gesteigerter Erregbarkeit nicht eher wieder eine Absenkung derselben zu erwarten wäre. Interessant wäre auch zu wissen, ob ein linearer Zunahme der Dauer auch eine lineare Zunahme der MEP-Amplitudenhöhe zu sehen wäre.

\subsection{Diskussion der Methodik}

Die Ergebnisse der hier vorliegenden Studie stammen aus Messungen eines kleinen Probandenkollektivs von 14 Probanden und können aufgrund dessen allenfalls eine Tendenz widerspiegeln.

Allerdings konnte durch wiederholte Sitzungen und Messungen derselben Probanden eine vergleichsweise hohe Datenmenge erhoben werden.

Für eine möglichst hohe Reliabilität wurden alle Messungen durch denselben Untersucher mit demselben Stimulationsgerät durchgeführt. Es wurde sich um eine möglichst hohe Konstanthaltung der äußeren Stimulationsbedingungen bemüht, die 
Probanden wurden gebeten, möglichst ausgeruht und entspannt, zu wenn möglich gleicher Tageszeit zu den Sitzungen zu kommen. Allerdings war es nicht möglich, sowohl äußere Einflüsse, wie beispielsweise unterschiedlicher Lärmpegel oder Temperaturunterschiede, wie auch unterschiedliche psychische und physische Verfassungen von Probanden und Untersucher absolut identisch zu reproduzieren. Hierdurch entstandene Effekte auf die Entstehung der Messergebnisse sind bei deren Betrachtung möglicherweise zu bedenken.

Zur Verbesserung der Objektivität wurde diese Studie außerdem als Einfachblindstudie durchgeführt, die Probanden waren unwissend hinsichtlich der Untersuchungsmethode. Weiterhin wurde der experimentelle Ablauf randomisiert, um eine höhere Validität zu erzielen.

Die Ausgangswerte der durch TMS-induzierten MEPs, die Baseline, wurden zwischen den Probanden verglichen. Es konnten keine signifikanten Unterschiede festgestellt werden. Das bedeutet für die hier vorliegenden Ergebnisse, dass das unterschiedliche Ausgangsniveau, gemessen an der Baseline, der zerebralen Erregung eine wenn überhaupt untergeordnete Rolle spielt.

Weiterhin waren Probanden mit psychiatrischen oder neurologischen Erkrankungen, die Pathologien im Bereich der zerebralen Erregung aufweisen könnten, von vornherein von der Studie ausgeschlossen. Dasselbe galt für Probanden, die jegliche Art der zentral sedierenden oder Bewusstseins verändernden Substanzen konsumierten.

Die Stimulationsbedingungen in Bezug auf Wellenlänge, Stärke der Stimulation und Art der Diodenbefestigung und -lokalisation wurden zur besseren Vergleichbarkeit denen der vorangegangenen Studie durch Chaieb et al. (2015) angepasst. So wurde lediglich die Dauer der Stimulation verändert, um eine möglichst sichere Aussage über den Einfluss 
der Stimulationsdauer auf die Veränderung der MEP-Amplitudenhöhe treffen zu können.

\subsection{Diskussion der Zielgrößenim Vergleich zu vorhergehenden Studien}

\subsubsection{Bisherige Ergebnisse experimenteller Studien}

Konstaninović et al. (2013) zeigten bereits die Auswirkungen 5-minütiger transkranieller Laserstimulation, allerdings ohne Plazebo-Kontrollgruppe. Dabei konnte gezeigt werden, dass

„TLS mit NIR-LLL eine transitorische Reduktion der Exzitabilität des stimulierten Kortex induziert" (Konstantinović et al. 2013).

Die Methodik war ähnlich der, die auch hier in der vorliegenden Studie verwendet wurde. Es wurde bei 14 Probanden für eine Dauer von 5 Minuten transkranieller Laser, allerdings pulsed wave, einer Wellenlänge von $905 \mathrm{~nm}$, also auch im Nahinfrarotbereich und auch über dem linken M1-Areal appliziert, mit 50mW/ $\mathrm{cm}^{2}$. Die MEP-Amplitudenhöhe wurde auch mittels TMS des FDI gemessen. Die MEP-Amplitudenhöhe wurde bis 30 Minuten nach der Stimulation gemessen und die jeweiligen MEPs zu den MEPAmplitudenhöhen der Baseline verglichen. In dieser Studie konnten sie, wie Chaieb et al. (2015) eine reduzierte MEP-Amplitudenhöhe innerhalb der ersten 20 Minuten nach transkranieller Laser-Stimulation zeigen.

Ergebnisse der von Chaieb et al. in der Klinik für klinische Neurophysiologie der Universität Göttingen durchgeführten Studie haben ergeben, dass eine 10minütige Stimulation mittels transkraniellem NIR-Laser in der Lage ist, die mittlere Amplitudenhöhe der durch TMS ausgelösten und gemessenen MEPs nach der Stimulation im Vergleich 
zur MEP- Amplitudenhöhe der Baseline zu senken (Chaieb et al. 2015). Dabei wurde dieselbe Methodik verwendet, wie auch in der von uns durchgeführten Studie. Verwendet wurde ebenfalls tNIRS, continuous wave, mit einer Stärke von $150 \mathrm{~mW}$ über einer Fläche von $0,35 \mathrm{~cm}^{2}$, was also einer Intensität von $500 \mathrm{~mW} / \mathrm{cm}^{2}$ entspricht. Insgesamt wurde eine Gesamtenergie von 1 Joule frei.

Die Studie ergab eine Verminderung der kortikalen Exzitabilität nach 10minütiger tNIRS von 20-30\%. Signifikant gesunkene Amplitudenhöhen der MEPs fanden sich 0 und 30 Minuten nach der Stimulation im Vergleich zur Baseline.

Zusammenfassend demonstrierten diese Ergebnisse, dass 10 Minuten anhaltende Stimulation mittels Infrarotlaser die kortikale Exzitabilität hemmt. Dabei hielt die hemmende Wirkung bis zu 30 Minuten nach der Stimulation an.

\subsubsection{Einsatz der Laser Therapie in klinischen Studien}

In Bezug auf einen möglichen klinischen Nutzen konnte bereits 2007 durch Lampl et al. in der NEST - 1 Studie (NeuroThera Effectiveness and Safety Trial) die „Sicherheit und Vorprüfung der Effektivität des NeuroThera Laser Systems in der Fähigkeit das 90-TagesOutcome bei Patienten mit ischämischen Schlaganfall zu verbessern“ (Lampl et al. 2007) gezeigt werden. Dabei hatten 120 Patienten in der groß angelegten internationalen multizentrischen Doppelblind-Studie Laser Therapie erhalten. Das Protokoll schrieb dabei Laser im Nahinfrarotbereich mit einer Wellenlänge von 808nm, also einer nahezu

gleichen Wellenlänge wie im hier vorliegenden Protokoll und Einsatz der Therapie innerhalb von 24 Stunden nach Einsetzen des Schlaganfall-Ereignisses. 20 vordefinierte Stellen wurden für je 2 Minuten pro Seite mit $1 \mathrm{~J} / \mathrm{cm}^{2}$ über der Hautoberfläche stimuliert. 
Die 2009 durch Zivin et al. durchgeführte NEST-2 Studie bestätigte unter Einhaltung der Stimulationsbedingungen der NEST-1 Studie die Sicherheit der Lasertherapie, konnte allerdings in Hinblick auf das verbesserte Outcome der Patienten und damit auf die Effektivität der Lasertherapie keine statistisch signifikanten Ergebnisse erzielen.

Die Effektivität der Lasertherapie sollte deshalb durch die randomisierte doppelblinde NEST- 3 Studie belegt werden (Zivin et al. 2014). Hierbei hatten 1000 Probanden teilnehmen sollen, welche die aktuelle medikamentöse Standardtherapie bei Schlaganfall erhalten werden und zusätzlich innerhalb 24 Stunden nach Schlaganfall Lasertherapie nach Protokoll wie in den vorhergegangenen NEST Studien erhalten sollen. Nach der Analyse von 566 Patienten wurde die Studie allerdings vorzeitig beendet, da kein Unterschied im Endresultat zwischen Patienten mit Plazebo-Therapie und Lasertherapie gesehen wurde. (Hacke et al. 2014) Dieses Ergebnis blieb konstant auch nach Einschließen und Auswerten aller 630 Patienten. Die Autoren fassten zusammen, dass „Lasertherapie keinen messbaren neuroprotektiven Effekt bei Patienten mit akutem ischämischen Schlaganfall hat" (Hacke et al. 2014).

\subsubsection{Diskussion der möglichen Ursachen einer Veränderung der kortikalen Exzitabilität}

Als Ursache für eine Hemmung der kortikalen Exzitabilität kommt möglicherweise die Verstärkung GABAerger Neurotransmission mit vermehrter Aktivierung hemmender interneuraler Netzwerke und deren Wirkung in Betracht oder aber die Verminderung glutamaterger Neurotransmission (Chaieb et al. 2014).

lonotrope Glutamatrezeptoren (NMDA - Rezeptoren) in der postsynaptischen Membran 
von Neuronen werden durch Ligandenbindung aktiviert und exzitatorische Potentiale (excitatory postsynaptic potentials, EPSPs) werden ausgelöst. Im Gegensatz dazu lösen GABA - Rezeptoren, besonders $\mathrm{GABA}_{\mathrm{A}}$, durch Erhöhung der Permeabilität für Cl-lonen inhibitorische Potentiale (inhibitory postsynaptic potentials, IPSPs) aus.

Chaieb et al. (2014) gaben als Ursache ihrer Ergebnisse eine „hochregulierte Atmung (ATP)“ an oder eine Downregulation der Mitochondrien selbst, sodass die normale Funktion GABAerger Neurotransmitter bereits ausreicht, die nicht-funktionierenden exzitatorischen Kreisläufe $\mathrm{zu}$ übertreffen und $\mathrm{zu}$ einer Hemmung zu führen. Konstantinovic et al. (2013) interpretierten ihre Ergebnisse als eine durch „low-level laser induzierte gesteigerte Resistenz gegenüber der durch TMS ausgelösten Depolarisation der kortikalen Pyramidenzellen" (Konstantinovic et al. 2013). Die Veränderungen der MEPs können also Änderungen der Membranexzitabilität der pyramidalen Neurone oder kortikalen Interneurone oder aber Veränderungen der synaptischen Wirksamkeit zwischen den einzelnen Neuronen bedeuten. Dabei führt die Applikation von transkranieller Laserstimulation durch Hemmung der Depolarisation des Ruhemembranpotentials der Neurone zu einer Verminderung der Exzitabilität.

\subsection{Veränderungen kortikaler Exzitabilität, hervorgerufen durch andere nicht-invasive Methoden}

\subsubsection{Stimulation durch tDCS (transcranial direct-current stimulation)}

Das menschliche Gehirn ist von außen stimulierbar und die kortikale Erregbarkeit beeinflussbar. Dies wurde bereits mit anderen Methoden erwiesen, so beispielsweise mittels transkranieller Stimulation mit Strom, tDCS (transcranial direct-current 
stimulation). Hierbei handelt es sich um Neurostimulation durch Elektroden mit konstantem niedrigem Strom. Nach tDCS konnten Veränderungen der kognitiven Funktionen nachgewiesen werden. (Nitsche et al. 2008). Nitsche und Paulus (2000) beispielsweise zeigten, dass Veränderungen der neuronalen Exzitabilität möglich sind, unterschiedlich je nach Polarität des applizierten Stroms.

\subsubsection{Abhängigkeit der Effekte von der Art der tDCS-Applikation}

Es kommt bei tDCS durch Anlage anodalen oder kathodalen Stroms zu unterschiedlichen Ergebnissen der Exzitabilitätsänderung des Gehirns.

Durch die Stimulation wird das Ruhemembranpotential der Neuronen de- oder hyperpolarisiert (Nitsche und Paulus 2000). Anodale Stimulation bewirkt eine Depolarisation des Ruhemembranpotentials und die neuronale Exzitabilität steigt. Hingegen bewirkt kathodale Stimulation eine Hyperpolarisation und die kortikale Exzitabilität sinkt (Nitsche, Seeber et al. 2005, Nitsche und Paulus 2000). Änderungen der Erregbarkeit des Gehirns sind also abhängig von der Polarität des Stroms und entsprechender Veränderung des Ruhemembranpotentials der Neuronen.

Außerdem konnten sie eine Abhängigkeit der Dauer der kortikalen Veränderung von der Dauer der Stromapplikation nachweisen.

Nitsche und Paulus (2000) zeigten eine Steigerung der MEP-Amplitudenhöhe nach anodaler tDCS und die unterschiedlichen Ergebnisse je nach Dauer der Stimulation. In ihrer Studie wurden die Elektroden über dem Areal des rechten abductor Digiti minimi (ADM) und der kontralateralen Orbita platziert. Es wurde kontinuierlich fließender Strom über 1-5 Minuten appliziert, mit 0,2-1,0 mA. 
Dabei wurde in verschiedenen Experimenten der Stromfluss als Variable verändert, die Dauer oder die Art des Stroms (kathodal oder anodal). In einem Experiment wurden Stromstärken von 0,2 - $1 \mathrm{~mA}$ appliziert bei gleichbleibender Stimulationsdauer von 5 Minuten und nur anodaler Stimulation. In einem anderen Experiment wiederum wurde bei gleichbleibender Stromstärke von 1mA die Dauer variiert von 1 - 5 Minuten Stimulation. Im letzten Experiment wurde anodaler und kathodaler Strom verglichen bei gleichbleibender Dauer von 5 Minuten und Fluss von $1 \mathrm{~mA}$.

Dabei zeigten sie, dass zur Induktion von Nacheffekten eine Dauer von mindestens 3 Minuten nötig ist und die Steigerung der Intensität oder der Dauer zu längeren und größeren Nacheffekten der Stimulation führt.

\subsubsection{Abhängigkeit der Effekte von der Dauer der tDCS-Applikation}

Langzeitpotenzierung (englisch: long-term potentiation, LTP) ist ein wichtiger Mechanismus des Lernens. Dabei ist es ein komplexer Prozess, bestehend unter anderem aus anhaltendem Gebrauch oder Nichtgebrauch und Training von Synapsen und synaptischen Verbindungen (Feldmann 2009). So ist ein grundlegender Mechanismus der Langzeitpotenzierung eine verlängerte Verstärkung der Aktivität neuronaler Synapsen. Monte-Silva et al. (2012) erzeugten durch periodisch repetitiv angewandte tDCS-Stimulation einen Effekt, welcher wie LTP einen länger anhaltenden Effekt der tDCS-Stimulation am Gehirn auch nach Beendigung der Stimulation erzeugte. Sie postulierten, dass Veränderungen der Exzitabilität während der Applikation von tDCS durch Veränderung des Ruhemembranpotentials hervorgerufen werden, wohingegen die länger anhaltenden Nacheffekte durch NMDA-Rezeptor-abhängige Exzitabilitätsveränderungen entstehen. 
Monte-Silva et al. applizierten über 13 Minuten anodale tDCS mit 1,0mA über dem M1Areal der rechten Hand. Danach applizierten sie entweder direkt ohne Pause erneut 13 Minuten tDCS nach einer Pause von 3 Minuten, nach einer Pause von 20 Minuten, nach einer Pause von 3 Stunden oder nach einer Pause von 24 Stunden.

Als Ergebnis konnten sie zeigen, dass wiederholte tDCS in der Lage ist, länger anhaltende kortikale Veränderungen zu erzeugen, wenn die erneute Stimulation in die Zeit der Nacheffekte der ersten Stimulation fällt, was im Fall der Studie nur für die Pause von 3 bis 20 Minuten zutraf. Eine längere Pause zwischen zwei aufeinanderfolgenden Stimulationen verringerte die Nacheffekte der tDCS.

Außerdem wiesen Monte-Silva et al. wie Nitsche et al. (2003) durch Gabe eines NMDARezeptorblockers die Abhängigkeit der länger anhaltenden Nacheffekte von NMDARezeptoren nach (siehe unten).

Als Ursache der gesteigerten Exzitabilität bei längerer Stimulationsdauer vermuten Monte- Silva et al. die Aktivierung hyperpolarisierender Kaliumkanäle durch erhöhte intraneurale Kalziumkonzentrationen, welche durch länger andauernde Depolarisation geöffnet bleiben. Interessant ist hier auch, dass wenn man einen Kalziumkanal-Blocker gibt, der tDCS - Effekt ausbleibt, was die genannte Theorie stützt.

Die in der hier durchgeführten Studie vorliegenden Ergebnisse einer gesteigerten kortikalen Erregbarkeit nach längerer Stimulation mittels transkraniellen Lasers von 20 Minuten unterstützen diese These und suggerieren, dass auch durch tNIRS hyperpolarisierende Kaliumkanäle aktiviert werden.

In einer anderen Studie untersuchten Batsikadze et al. (2013) unterschiedliche Intensitäten des applizierten Stroms und stimulierten mit $2 \mathrm{~mA}$, statt wie Nitsche und Paulus (2000) mit 1 mA, über eine Dauer von 20 Minuten. Dabei konnten sie zeigen, dass es durch die erhöhte Intensität des applizierten Stroms zu einer Umkehr der 
induzierten Exzitabilitätsveränderung kommt. In den Experimenten von Nitsche und Paulus (2000) wurde durch kathodalen Strom bei einer Intensität von $1 \mathrm{~mA}$, appliziert über 20 Minuten eine Verringerung der neuronalen Erregbarkeit verursacht. Bei Batsikadze et al. (2013) kam es zu einer Steigerung nach 20 Minuten und Stimulation mit 2 mA. Als Ursache für diese Veränderung postulierten Batsikadze et al. (2013) wie Monte-Silva et al. (2012) einerseits erhöhte intraneuronale Kalziumkonzentrationen, die zu einer Richtungsänderung der Exzitabilitätsveränderung führen könnten. Durch höhere Kalziumkonzentrationen, verursacht durch intensiveren Strom, kommt es eher zu Langzeitpotenzierung (LTP, Cho et al. 2001) und damit zu anhaltenden Nacheffekten nach Stimulation.

„Es gibt zwingende Beweise aus Experimenten in neuronalen Kulturen, dass zentrale Neurone in der Lage sind, durchschnittliche neuronale Feuerungsraten um einen homöostatischen Sollwert aufrecht zu erhalten. [...], wenn neuronales Feuern über die Zeit reduziert ist, kompensieren [dies] die Neurone und das Feuern ist wiederhergestellt.“ (Turrigiano 2012)

Im Sinne dieser homöostatischen Gegenregulierung wäre durch synaptische Plastizität, beispielsweise durch postsynaptische Rezeptorakkumulation (Turrigiano 2008), eine Hochregulierung synaptischer Verbindungen denkbar, um der von außen induzierten Hemmung entgegenzuwirken und einen eigenen Normwert an Aktivität der Neurone wiederzuerlangen.

Gina Turrigiano (2012) führt auch aus, dass im Rahmen der synaptischen Plastizität Veränderungen der Feuerungsrate eines Neurons durch dessen kalziumabhängige Sensoren entdeckt werden und kompansatorisch Glutamatrezeptoren hoch- oder herunterreguliert werden können, um die Aktivität eines Neurons um einen Sollwert 
konstant halten zu können, auch Homöostase genannt. Das könnte ein Erklärungsansatz für die Ergebnisse der hier vorliegenden Studie sein, in denen die hemmende Wirkung einer 10 minütigen - tDCS nicht anhält oder steigt mit verlängerter Dauer, sondern eher einen gegenteiligen Effekt hervorruft.

\subsection{Ausblick und Limitationen}

Es konnte gezeigt werden, dass die Stimulationsdauer mit NIR-Laser einen entscheidenden Einfluss auf die Modulierbarkeit der Erregbarkeit des Gehirns hat. Während eine 10-minütige Stimulationszeit eine Verminderung der kortikalen Exzitabilität bewirkt (Chaieb et al. 2015), verstärkt eine doppelte Stimulationsdauer von 20 Minuten die kortikale Exzitabilität.

Eine ideale Stimulationsdauer scheint möglicherweise unter konstant gehaltenen anderen Stimulationsparametern zwischen diesen beiden Zeiten zu liegen, um weder eine hemmende, noch eine übermäßig steigernde Wirkung hervorzurufen. Interessant wären aber auch weitere Beobachtungen, ob mit noch längerer Stimulationsdauer nach Erreichen eines Maximums der Erregbarkeit vielleicht wieder eine Hemmung derselben folgt. Vielleicht wäre auch dort eine ideale Stimulationsdauer zur Veränderung kortikaler Exzitabilität zu finden.

Die Bestätigung der Ergebnisse und sicherere Aussagen müssten mit Hilfe weitergehender größer angelegter Untersuchungen getroffen werden, die aufgrund des intensiven Aufwands jedoch schwerer durchführbar sind.

Zur weiteren Erhöhung der Validität könnte eine weiterführende Studie als Doppel-BlindStudie durchgeführt werden und die Ergebnisse mit den hier vorliegenden verglichen 
werden.

Weiterhin sollten die Nacheffekte genauer untersucht werden beziehungsweise die Möglichkeit der Verlängerung der Effekte, beispielsweise durch Gabe von Medikamenten. Bisher waren die Effekte nach 20-minütiger Stimulationsdauer nur bis zu 30 Minuten nach Stimulationsende durch MEP-Veränderungen nachweisbar.

Pharmakologische Studien mit Gabe von NMDA-Rezeptoragonisten könnten möglicherweise zu einer länger anhaltenden gesteigerten Exzitabilität führen. So könnten auch die Ursachen der unterschiedlichen Exzitabilitätsveränderungen genauer untersucht werden.

Dann könnte möglicherweise durch zusätzliche Gabe von Rezeptor stimulierenden beziehungsweise blockenden Medikamenten eine gezielte Veränderung des Membranpotentials ausgelöst werden und so die Effekte von tNIRs verstärkt, beziehungsweise verlängert werden.

Bei optimalen Stimulationsbedingungen birgt die tNIRS so möglicherweise das Potential des klinischen Einsatzes und die Möglichkeit der Therapie im neurologischen Bereich, beispielsweise für Schlaganfallpatienten. Aus diesem Grund sollten weitergehende Untersuchungen folgen, diese optimalen Stimulationsbedingungen zu ergründen. 


\section{Zusammenfassung}

„Ernsthafte lebensbedrohliche Krankheiten, wie Schlaganfall, Herzinfarkt, Rückenmarksverletzung und traumatische Hirnverletzungen könnten bald für low-levellaser therapy zugänglich sein." (Chung et al. 2012).

Das Gehirn ist durch neuronale Plastizität in der Lage sich ständig zu verändern und neuen äußeren Umständen anzupassen, was die Grundlage für beispielsweise Lernvorgänge bildet. In dieser neuronalen Plastizität verbirgt sich damit auch ein großes Potential für die Medizin. Besonders Schlaganfallpatienten könnten von einer gesteuerten Modulation und Regeneration des Gehirns profitieren, wie bereits in vorhergehenden Studien gezeigt werden konnte (Oron et al. 2006, Litscher und Litscher 2013). Aus diesem Grund ist die gezielte Beeinflussbarkeit der neuronalen Plastizität von extern aktuell Gegenstand intensiver Forschung.

Eine Möglichkeit der Modulierbarkeit der neuronalen Plastizität ist der Einsatz der transkraniellen Infrarotlaserstimulation (tNIRS). Diese Methode könnte sich aufgrund ihrer Nicht-Invasivität und Schmerzfreiheit sowie der guten Steuerbarkeit durch den Behandelnden gut für den klinischen Einsatz am Patienten eignen. Deshalb wird diese Methode momentan in verschiedenen Studien untersucht und hinsichtlich ihrer unterschiedlichen Parameter getestet.

Zusammenfassend lässt sich sagen, dass die Dauer einer Stimulation mit tNIRS einen wichtigen Parameter bezüglich der Effekte von tNIRS auf die Modulierbarkeit der kortikalen Exzitabilität darstellt.

Eine Stimulation mit tNIRS von 10 Minuten wirkt hemmend auf die Exzitabilität des Gehirns. Eine kürzere Stimulationsdauer mit tNIRS von 5 Minuten wirkt geringfügig 
hemmend auf die kortikale Exzitabilität und zeigt nur eine mäßige Reduktion der MEPAmplitudenhöhe nach Stimulation im Vergleich zur Baseline.

Eine längere Stimulationsdauer von 20 Minuten jedoch wirkt aktivierend auf die kortikale Exzitabilität und erhöht die MEP-Amplitudenhöhe nach Stimulation signifikant bis zu 30 Minuten nach Stimulationsende.

Die vorliegende Studie hat ergeben, dass die optimale Stimulationsdauer zur Modulierbarkeit der kortikalen Exzitabilität zwischen 10 Minuten und 20 Minuten Stimulationsdauer zu liegen scheint.

Zur genaueren Abklärung sollten hierfür weitergehende Untersuchungen stattfinden. Wenn die Möglichkeit der Modulation der kortikalen Exzitabilität weiterhin Gegenstand aktueller Forschung bleibt, wäre es in der Zukunft vielleicht möglich, eine neue Therapiemöglichkeit für ernsthafte lebensbedrohliche Erkrankungen zu finden und im klinischen Alltag zu etablieren. 


\section{Literaturverzeichnis}

- Amassian VE, Stewart M, Quirk GJ, Rosenthal JL (1987): Physiological basis of motor effects of a transient stimulus to cerebral cortex. Neurosurgery 20,74-93

- Anand S, Hotson J (2002): Transcranial magnetic stimulation: neurophysiological applications and safety. Brain Cogn $\underline{50}, 366-386$

- Barker AT (1999): The history and basic principles of magnetic nerve stimulation. Electroencephalogr Clin Neurophysiol 51, 3-21

- Barker AT, Jalinous R, Freeston IL (1985): Non-invasive magnetic stimulation of human motor cortex. Lancet 19851 (8437), 1106-1107

- Batsikadze G, Moliadze V, Paulus W, Kuo MF, Nitsche MA (2013): Partially non-linear stimulation intensity-dependent effects of direct current stimulation on motor cortex excitability in humans. J Physiol $\underline{591}, 1987-$ 2000

- Brandt SA, Ploner CJ, Meyer BU (1997): Repetitive transkranielle Magnetstimulation. Möglichkeiten, Grenzen und Sicherheitsaspekte. Nervenarzt $\underline{68}, 778-784$

- Chaieb L, Antal A, Masurat F, Paulus W (2015): Neuroplastic effects of transcranial near- infrared stimulation (tNIRS) on the motor cortex. Front Behav Neurosci $\underline{9}, 147$

- Cho K, Aggleton JP, Brown MW, Bashir ZI (2001): An experimental test of the role of postsynaptic calcium levels in determining synaptic strength using perirhinal cortex of rat. The Journal of physiology $\underline{532}, 459-466$

- Chow RT, Johnson MI, Lopes-Martins RAB, Bjordal JM (2009): Efficacy of lowlevel laser therapy in the management of neck pain: a systematic review and meta-analysis of randomised placebo or active-treatment controlled trials. Lancet $\underline{375}, 894$

- $\quad$ Chung H, Dai T, Sharma SK, Huang Y-Y, Carroll JD, Hamblin MR (2012): The nuts and bolts of low-level laser (light) therapy. Ann Biomed Eng 40, 516-533

- Deklaration von Helsinki (18. WMA-Generalversammlung (1964), aktuelles Amendement: 64. WMA-Generalversammlung (2013) Fortaleza, Brasilien)

- DeTaboada L, Ilic S, Leichliter-Martha S, Oron U, Oron A, Streeter J (2006): Transcranial application of low-energy laser irradiation improves neurological deficits in rats following acute stroke. Lasers Surg Med $\underline{38}, 70-73$

- Eells JT, Henry MM, Summerfelt P, Wong-Riley, MT, Buchmann EV, Kane M, Whelan NT, Whelan HT (2003): Therapeutic photobiomodulation for methanolinduced retinal toxicity. Proc Natl Acad Sci USA 100, 3439-3444 
- Ekstrand MI, Terzioglu M, Galter D, Zhu S, Hofstetter C, Lindqvist E, Thams S, Bergstrand A, Hansson FS, Trifunovic A, Hoffer B, Cullheim S, Mohammed AH, Olson L, Larsson NG (2007): Progressive parkinsonism in mice with respiratorychain-deficient dopamine neurons. Proc Natl Acad Sci USA 104, 1325-1330.

- Ellaway PH, Davey NJ, Maskill DW, Rawlinson SR, Lewis HS, Anissimova NP (1998): Variability in the amplitude of skeletal muscle responses to magnetic stimulation of the motor cortex in man. Electroencephalogr Clin Neurophysiol. $\underline{109}, 104-113$

- Eriksson PS, Perfilieva E, Björk-Eriksson T, Alborn AM, Nordborg C, Peterson DA, Gage FH (1998): Neurogenesis in the adult human hippocampus. Nat Med 4, 1313-1317.

- Feldman DE (2009): Synaptic Mechanisms for Plasticity in Neocortex. Annu Rev Neurosci $\underline{32}, 33-55$.

- Galvan V, Jin K (2007): Neurogenesis in the aging brain. Clin Interv Aging2, 605-610.

- Greco M, Guida G, Perlino E, Marra E, Quagliariello E (1989): Increase in RNA and protein synthesis by mitochondria irradiated with helium-neon laser. Biochem Biophys Res Commun 163, 1428-1434

- Hacke W, Schellinger PD, Albers GW, Bornstein NM, Dahlof BL, Fulton R, Kasner SE, Shuaib A, Richieri SP, Dilly SG, Zivin J, Lees KR, NEST-3 Committees and Investigators (2014): Transcranial laser therapy in acute stroke treatment: results of neurothera effectiveness and safety trial 3 , a phase III clinical end point device trial. Stroke $\underline{45}, 3187-3193$

- Hallett M (2000): Transcranial magnetic stimulation and the human brain. Nature $\underline{406}, 147-150$

- Hashmi JT, Huang Y-Y, Osmani BZ, Sharma SK, Naeser MA, Hamblin MR (2010): Role of low-level laser therapy in neurorehabilitation. PM \& R $\underline{2}, 292$ 305

- Karu T (1989 a): Laser biostimulation: a photobiological phenomenon. J Photochem Photobiol B. $\underline{3}, 638-640$

- Karu T (1989 b): Photobiology of low-power laser effects. Health Phys $\underline{56}, 691-704$

- Karu T (1999): Primary and secondary mechanisms of action of visible to near-IR radiationon cells. J Photochem Photobiol B $\underline{49}, 1-17$

- Karu T, Pyatibrat L, Kalendo G (1995): Irradiation with He-Ne laserincreases ATP level in cells cultivated in vitro. J Photochem Photobiol B 27,219-223

- Kiers L, Cros D, Chiappa KH, Fang J (1993): Variability of motor potentials evoked by transcranial magnetic stimulation. Electroencephalogr Clin Neurophysiol $\underline{89}, 415-423$

- Konstantinović LM, Jelić MB, Jeremić A, Stevanović VB, Milanović SD, Filipović SR (2013): Transcranial application of near-infrared low-level laser can modulate 
cortical excitability. Lasers Surg Med $\underline{45}$, 648-653

- Lampl Y, Zivin JA, Fisher M, Lew R, Welin L, Dahlof B, Borenstein P, Andersson B, Perez J, Caparo C, llic S, Oron U (2007): Infrared laser therapy for ischemic stroke: a new treatment strategy: results of the NeuroThera Effectiveness and Safety Trial-1 (NEST-1). Stroke $\underline{38}, 1843-1849$

- Lapchak PA (2010): Taking a light approach to treating acute ischemic stroke patients: transcranial near-infrared laser therapy translational science. Ann Med 42, 576-586

- Lapchak PA, DeTaboada L (2010): Transcranial near infrared laser treatment (NILT) increases cortical adenosine-5'-triphosphate (ATP) content following embolic strokes in rabbits. Brain Res $\underline{1306}, 100-105$

- Lapchak PA, Wei J, Zivin JA (2004): Transcranial infrared laser therapy improvesclinical rating scores after embolic strokes in rabbits. Stroke $\underline{35}$, 1985-1988

- Lapchak PA, Salgado KF, Chao CH, Zivin JA (2007): Transcranial near-infrared light therapy improves motor function following embolic strokes in rabbits: an extended therapeutic window study using continuous and pulse frequency delivery modes. Neuroscience 1묘, 907-914

- Lazarov O, Marr RA (2010): Neurogenesis and Alzheimer's disease: at the crossroads. Exp Neurol 223, 267-281

- Litscher D, Litscher G (2013): Laser Therapy and Stroke: Quantification of Methodological Requirements in Consideration of Yellow Laser. International Journal of Photoenergy $\underline{6}, 1-4$

- Litscher G, Liu C-Z, Wang L, Wang L-P, Li Q-Q, Shi G-X, Gaischek I, Litscher D, Wang X-M (2013): Improvement of the dynamic responses of heart rate variability patterns after needle and laser acupuncture treatment in patients with burnout syndrome: a transcontinental comparative study. Evid Based Complement Alternat Med 2013, 128721

- Mester E, Spiry T, Szende B, Tota JG (1971): Effect of laser rays on wound healing. Am J Surg 122, 532-535

- Mills KR, Murray NM, Hess CW (1987): Magnetic and electrical transcranial brain stimulation: physiological mechanisms and clinical applications.

Neurosurgery $\underline{20}, 164-168$

- Monte-Silva K, Kuo MF, Hessenthaler S, Fresnoza S, Liebetanz D, Paulus W, Nitsche MA (2012): Induction of late LTP-like plasticity in the human motor cortex by repeated non- invasive brain stimulation. Brain Stimul $\underline{6}, 424-432$

- Naeser MA (1997): Neurological rehabilitation: acupuncture and laser acupuncture to treat paralysis in stroke, other paralytic conditions and pain in carpal tunnel syndrome. JAltern Complement Med $\underline{3}$, 425-428

- Naeser MA, Saltmarche A, Krengel MH, Hamblin MR, Knight JA (2011): Improved cognitive function after transcranial, light-emitting diode treatments in chronic, 
traumatic brain injury: two case reports. Photomed Laser Surg 29 , 351-358

- Nitsche MA, Paulus W (2000): Excitability changes induced in the human motor cortex by weak transcranial direct current stimulation. J Physiol $\underline{527}, 633-639$

- Nitsche MA, Fricke K, Henschke U, Schlitterlau A, Liebetanz D, Lang N, Henning S, Tergau F, Paulus W (2003): Pharmacological modulation of cortical excitability shifts induced by transcranial direct current stimulation in humans. $J$ Physiol 553, 293-301

- Nitsche MA, Seeber A, Frommann K, Klein CC, Rochford C, Nitsche MS, Fricke K, Liebetanz D, Lang N, Antal A, Paulus W, Tergau F (2005): Modulating parameters of excitability during and after transcranial direct current stimulation of the human motor cortex. J Physiol $\underline{568}, 291-303$

- Nitsche MA, Cohen LG, Wassermann EM, Priori A, Lang N, Antal A, Paulus W, Hummel F, Boggio PS, Fregni F, Pascual-Leone A (2008): Transcranial direct current stimulation: State of the art 2008. Brain Stimul 1, 206-223

- Oron A, Oron U, Chen J, Eilam A, Zhang C, Sadeh M, Lampl Y, Streeter J, DeTaboada L, Chopp M (2006): Low-level laser therapy applied transcranially to rats after induction of stroke significantly reduces long-term neurological deficits. Stroke 37,2620-2624

- Oron A, Oron U, Streeter J, DeTaboada L, Alexandrovich A, Trembovler V, Shohami E (2007): low-level laser therapy applied transcranially to mice following traumatic brain injury significantly reduces long-term neurological deficits. J Neurotrauma 24, 651-656

- Oron A, Oron U, Streeter J, DeTaboada L, Alexandrovich A, Trembovler V, Shohami E (2012): Near infrared transcranial laser therapy applied at various modes to mice following traumatic brain injury significantly reduces long-term neurological deficits. J Neurotrauma $\underline{29}, 401-407$

- Pascual-Leone A, Houser CM, Reese K, Shotland LI, Grafman J, Sato S, VallsSolé J, Brasil- Neto JP, Wassermann EM, Cohen LG (1993): Safety of rapid-rate transcranial magnetic stimulation in normal volunteers. Electroencephalogr Clin Neurophysiol $\underline{89}, 120-130$

- Passarella S, Casamassima E, Molinari S, Pastore D, Quagliariello E, Catalano IM, Cingolani A (1984): Increase of proton electrochemical potential and ATP synthesis in rat liver mitochondria irradiated in vitro by helium-neon laser. FEBS Lett $\underline{175}$, 95-99

- Pastore D, Greco M, Petragallo VA, Passarella S (1994): Increase in --H+/eratio of the cytochrome $\mathrm{c}$ oxidase reaction in mitochondria irradiated with helium-neon laser. Biochem Mol Biol Int 34, 817-826

- Ravnborg M, Dahl K (1991): Examination of central and peripheral motor pathways by standardized magnetic stimulation. Acta Neurol Scand $\underline{84}$, 491-497 
- Rojas JC, Gonzalez-Lima F (2013): Neurological and psychological applications of transcranial lasers and LEDs. Biochem Pharmacol $\underline{86}$, $447-457$

- Rossini PM, Pauri F (2000): Neuromagnetic integrated methods tracking human brain mechanisms of sensorimotor areas 'plastic' reorganisation. Brain Res Brain Res Rev $\underline{33}$, 131-154

- Schapira AH (2012): Mitochondrial diseases. Lancet $\underline{379}$, 1825-1834

- Sommer AP, Bieschke J, Friedrich RP, Zhu D, Wanker EE, Fecht HJ, Mereles D, Hunstein W (2012): 670nm laser light and EGCG complementarily reduce amyloid- $\beta$ aggregates in human neuroblastoma cells: basis for treatment of Alzheimer's disease? Photomed Laser Surg 30, 54-60

- Stemer AB, Huisa BN, Zivin JA (2010): The evolution of transcranial laser therapy for acute ischemic stroke, including a pooled analysis of NEST-1 and NEST-2. Curr Cardiol Rep 12, 29-3

- The National Institute of Neurological Disorders and Stroke rt-PA Stroke Study Group (1995): Tissue plasminogen activator for acute ischemic stroke. N Engl J Med $\underline{333}, 1581-1587$

- Thom T, Haase N, Rosamond W, Howard VJ, Rumsfeld J, Manolio T, Zheng Z-J, Flegal K, O'Donnell C, Kittner S, et al. (2006): Heart disease and stroke statistics--2006 update: a report from the American Heart Association Statistics Committee and Stroke Statistics Subcommittee. Circulation 113, e85-151

- Turrigiano GG (2008): The self-tuning neuron: synaptic scaling of excitatory synapses. Cell $\underline{135}, 422-435$

- Turrigiano G (2012): Homeostatic synaptic plasticity: local and global mechanismsfor stabilizing neuronal function. Cold Spring Harb Perspect Biol 4, a005736

- Wassermann EM (1998): Risk and safety of repetitive transcranial magnetic stimulation: report and suggested guidelines from the International Workshop on the Safety of Repetitive Transcranial Magnetic Stimulation, June 5-7, 1996. Electroencephalogr Clin Neurophysiol 108, 1-16

- Wassermann EM, Grafman J, Berry C, Hollnagel C, Wild K, Clark K, Hallett M (1996): Use and safety of a new repetitive transcranial magnetic stimulator. Electroencephalogr Clin Neurophysiol 101, 412-417

- Weber M (2005): Nadeln aus Licht - Vorstellung einer neuen Therapiemethode. DZA 48, 24-32.

- Weber M , Fussgänger-May T, Wolf T (2007): "Needles of Light": A New Therapeutic Approach. Medical Acupuncture 19, 141-151.

- Zivin JA, Albers GW, Bornstein N, Chippendale T, Dahlof B, Devlin T, Fisher M, Hacke W, Holt W, llic S et al. (2009): Effectiveness and safety of transcranial laser therapy for acute ischemic stroke. Stroke $\underline{40}, 1359-1364$. 
- Zivin JA, Sehra R, Shoshoo A, Albers GW, Bornstein NM, Dahlof B, Kasner SE, Howard G, Shuaib A, Streeter J, Richieri SP, Hacke W, NEST-3 investigators (2014): NeuroThera ${ }^{\circledR}$ Efficacy and Safety Trial-3 (NEST-3): a double-blind, randomized, sham-controlled, parallel group, multicenter, pivotal study to assess the safety and efficacy of transcranial laser therapy with the NeuroThera ${ }^{\circledR}$ Laser System for the treatment of acute ischemic stroke within $24 \mathrm{~h}$ of stroke onset. Int J Stroke $\underline{9}, 950-955$. 
